\title{
Convection in the atmospheres and envelopes of Pre-Main Sequence stars
}

\author{
J. Montalbán ${ }^{1,2}$, F. D’Antona ${ }^{2}$, F. Kupka ${ }^{3, \star}$, and U. Heiter ${ }^{4}$ \\ ${ }^{1}$ Institute of Astrophysics and Geophysics, University of Liège, 17, allée du 6-Août, Bât. B5c, 4000 Liège, Belgium \\ 2 INAF, Osservatorio Astronomico di Roma, 00040 Monteporzio, Italy \\ e-mail: dantona@mporzio.astro.it \\ 3 Astronomy Unit, School of Mathematical Sciences, Queen Mary, University of London, Mile End Road, \\ London E1 4NS, UK \\ e-mail: fk@mpa-garching-mpg.de \\ ${ }^{4}$ Department of Astronomy and Space Physics, Uppsala University, Box 515, 75120 Uppsala, Sweden \\ e-mail: ulrike@astro.uu.se
}

Received 21 February 2003 / Accepted 9 December 2003

\begin{abstract}
The $T_{\text {eff }}$ location of Pre-Main Sequence (PMS) evolutionary tracks depends on the treatment of over-adiabaticity (D'Antona \& Mazzitelli 1994, 1998). Since the convection penetrates into the stellar atmosphere, also the treatment of convection in the modeling of stellar atmospheres will affect the location of the Hayashi tracks. In this paper we present new non-grey PMS tracks for $T_{\text {eff }}>4000 \mathrm{~K}$. We compute several grids of evolutionary tracks varying: $i$ ) the treatment of convection: either the Mixing Length Theory (MLT) or Canuto et al. (1996, CGM) formulation of a Full Spectrum of Turbulence; ii) the atmospheric boundary conditions: we use the new Vienna grids of ATLAS9 atmospheres (Heiter et al. 2002a), which were computed using either MLT (with $\alpha=\Lambda / H_{\mathrm{p}}=0.5$ ) or CGM treatments. For comparison, we also compute grids of models with the NextGen (Allard \& Hauschildt 1997, AH97) atmosphere models, and a $1 M_{\odot}$ grey MLT evolutionary track using the $\alpha$ calibration based on 2D-hydrodynamical models (Ludwig et al. 1999). These different grids of models allow us to analyze the effects of convection modeling on the non-grey PMS evolutionary tracks. We disentangle the effect of the wavelength dependent opacity on a self-consistent treatment of convection in the atmosphere from the role of the convection model itself in the atmosphere and in the interior. While for some parts of the HR diagram (e.g., A stars) a low efficiency of atmospheric convection is clearly indicated by the data, for others the evidence is conflicting, showing the weaknesses of all the presently adopted local convection models. Nevertheless, the assumption of a low photospheric efficiency permits us to reproduce a larger amount of data and we have hence restricted our study to this case and draw the following conclusions for it: $i$ ) in spite of the solar calibration, if MLT convection is adopted a large uncertainty results in the shape and location of PMS tracks, and the MLT calibration loses sense. ii) As long as the model of convection is not the same in the interior and in the atmosphere, the optical depth at which we take the boundary conditions is an additional parameter of the models. iii) Furthermore, very different sub-atmospheric structures are obtained (for MS and PMS stellar models) depending not only on the treatment of convection, but also on the optical depth at which the boundary conditions are taken. iv) The comparison between NextGen based models and ATLAS9 based models shows that in the $T_{\text {eff }}$ domain they have in common $(4000-10000 \mathrm{~K})$ the improved opacities in NextGen atmosphere models have no relevant role on the PMS location, this being determined mainly by the treatment of the over-adiabatic convection. v) In the framework of standard stellar modeling (i.e., non-magnetic, non-rotating, spherical models), the comparison between theoretical models and observational data in very young binary systems indicates that, for both treatments of convection (MLT and CGM) and for any of the atmosphere grids (including those based on the 2D-hydrodynamical atmosphere models), the same assumption for convection cannot be used in PMS and MS: either the models fit the MS - and the Sun in particular - or they fit the PMS. Convection in the PMS phase appears to be less efficient than what is necessary to fit the Sun.
\end{abstract}

Key words. stars: evolution - stars: atmospheres - stars: pre-main sequence - convection

\section{Introduction}

The experimental data presently available for young objects in star-formation regions need to be compared with theoretical

Send offprint requests to: J. Montalbán,

e-mail: j.montalban@ulg.ac.be

^ Now at Max-Planck-Institute for Astrophysics, KarlSchwarzschild Str. 1, 85741 Garching, Germany. evolutionary tracks to be correctly interpreted in terms of age, mass and chemical composition. As discussed in D'Antona (2000) and the references therein, the HR diagram location of a star during its Pre-Main-Sequence (PMS) evolution is very sensitive to physical inputs such as low-temperature opacity, equation of state, rotation, atmosphere model, and convection treatment. 
During the last years, a lot of work has been done to improve the knowledge of low-temperature opacities and to include them in the modeling of stellar atmospheres. The variety of theoretical evolutionary tracks available today (new convection treatment with grey boundary conditions; classic convection with non-grey atmosphere models...) has contributed to creating some confusion about the effect of the different physical inputs on the results. Our aim is to extricate the different roles of non-grey atmospheres and of convection in evolutionary track computations. As shown by Montalbán et al. (2001), this can be done only using models with a self-consistent treatment of convection both in the atmosphere and in the interior.

In stars on the cool side of the HR diagram, convection is deep and defines the "envelope" portion of the star in which the dominant mode of energy transport is convection. In particular, Pre-Main Sequence (PMS) models are largely convective and - due to the low gravity and low temperature - the convection can be over-adiabatic in extended regions of the stars. Consequently, the shape of the corresponding evolutionary tracks depends on the efficiency of the convective transport of energy.

The convective zone increases with decreasing $T_{\mathrm{eff}}$ and it is gradually shifted towards larger depths, so that the structure of the surface layers is less and less affected by the convection as $T_{\text {eff }}$ decreases. For temperatures lower than $4700 \mathrm{~K}$, however, the convection zone rises again due to the dissociation of $\mathrm{H}_{2}$. On the other hand, for given $T_{\text {eff }}$ and metallicity, and decreasing gravity, the convective flux decreases because of the lower density and, therefore, the over-adiabatic region in the atmosphere is more extended. Consequently, in cool stars, a large part of the photosphere actually forms the uppermost portion of the convective envelope.

Several facts indicate that the classical grey atmosphere approximation adopted in stellar computation is not valid for cool stars: the diffusion approximation is not valid up to $\tau=$ 2/3 (Morel et al. 1994), and the convective transport of energy has to be taken into account in the atmosphere as well. Furthermore, the introduction of the frequency dependence of opacity modifies the onset of convection, and the grey approximation produces errors in the theoretical $T_{\text {eff }}$ and in the colors of low-mass stars (Baraffe et al. 1995; Baraffe \& Chabrier 1997). Therefore, in the computation of stellar models, the boundary conditions (BCs) at the surface must be provided by non-grey modeling of stellar atmospheres, taking into account the dependence of opacity on frequency and an adequate treatment of convection.

Unfortunately, for the time being, only very simple local convection models are available for routine computation of extended grids of model atmospheres, while detailed numerical simulations are still unaffordable for applications such as stellar evolution that requires the calculation of many thousands of individual model atmospheres over the HR diagram.

The "standard model" of convection in stellar evolution is the mixing length theory (MLT, Böhm-Vitense 1958), where turbulence is described by a relatively simple model that contains essentially one adjustable parameter, the mixing length: $\Lambda=\alpha H_{\mathrm{p}}\left(H_{\mathrm{p}}\right.$ being the local pressure scale height and $\alpha$ an unconstrained parameter). Canuto \& Mazzitelli (1991, CM) and
Canuto et al. (1996, CGM) made available an alternative model (Full Spectrum Turbulence - FST - models), that overcomes some of the problems of MLT, while keeping a low computational cost. The main characteristics and differences of these models are described in Sect. 2.

D'Antona \& Mazzitelli (1994, DM94) published four sets of PMS evolutionary tracks that made it possible to study the effect of low-temperature opacity and treatment of convection on stellar evolution. These tracks were computed with both kinds of convection treatments: MLT calibrated on the Sun, and the FST model with the CM formalism. The results showed that the $T_{\text {eff }}$ location of Hayashi tracks has a strong dependence on the treatment of convection. DM94's models, widely used and tested with observations, were revised in D'Antona \& Mazzitelli (1997, 1998 - DM97, 98) by introducing several improvements in the micro-physics (updated opacity tables and equation of state) and in the macro-physics (the updated FST treatment of convection by Canuto et al. 1996). The DM97 models were still employing grey atmospheric BCs, but the problem of matching convection in the interior and in the atmosphere occurs already in these models. In fact the DM97,98 models make only an exploratory approximation for defining the convective scale length if convection penetrates in the atmosphere, namely, they do not include the atmospheric convective depth in the computation of the scale. With this choice, convection in the sub-photospheric layers is less efficient, and very low mass stars are cooler by up to $\sim 150 \mathrm{~K}^{1}$. DM98 suggested that since the over-adiabaticity is present in low gravity/low temperature atmospheres, the FST convection treatment would modify convection also in the atmosphere, therefore it would be very important to include a revised treatment of convection in model atmospheres.

Recently, Heiter et al. (2002a) have published new atmosphere model grids based on Kurucz's ATLAS9 code (1993). They performed calculations for different treatments of nonadiabatic convection: MLT ( $\alpha=0.5), \mathrm{CM}$, and CGM (for a smaller range of parameters, MLT $-\alpha=1.25$ - was used as well). These choices for modeling convection are extensively motivated in Sect. 2 of that paper. As one of their main results, the authors conclude that MLT models with a small mixing length parameter (e.g., $\alpha \sim 0.5$ ) and FST models are equivalent in the atmospheric region where the observed flux originates. Both treatments predict a low convective efficiency for these layers. The deep atmospheric structures, however, are different, and each $T(\tau)$ relation represents stars which differ in radius and luminosity, hence the PMS tracks will also be different. Furthermore, if MLT is adopted, and a low $\alpha$-value is used in the atmosphere $\left(\alpha_{\mathrm{atm}}\right)\left(\alpha_{\mathrm{atm}}=0.5\right.$, as adopted in Heiter et al. 2002a, or $\alpha_{\text {atm }}=1$ as in Hauschildt et al. $1999 \mathrm{a}^{2}$ ), we must compensate, in order to fit the Sun, for the high

\footnotetext{
1 The procedure is extensively explained in DM98. Although this is the only case in which FST convection results in a lower global efficiency than MLT, this result has been ascribed in Baraffe et al. (2002) to the general behavior of FST large temperature gradients in the atmosphere.

2 NextGen atmosphere models named here AH97 were partially published in Hauschildt et al. (1999a).
} 
over-adiabaticity in the atmosphere, by using an $\alpha$-value in the interior $\left(\alpha_{\text {int }}\right.$ much larger than $\left.\alpha_{\text {atm }}\right)$. FST, by construction, reproduces this behavior: it is very inefficient at the outer boundary of the convection zone, and very efficient in the inner layers. As a consequence, FST has the advantage of simultaneously (and thus consistently) fitting, without arbitrary tuning of the parameter set $\left(\alpha_{\mathrm{atm}}, \tau_{\mathrm{ph}}, \alpha_{\mathrm{int}}\right)$, the Balmer line profiles (Heiter et al. 2002a) and the solar radius (Heiter et al. 2002b and this paper).

In order to analyze the impact of sub-photospheric convection on the HR location of the Hayashi tracks, we computed solar composition stellar models for masses from 0.6 to $2.0 M_{\odot}$ with several combinations of convection model and boundary conditions (Table 1). The physical inputs of these models are described in Sect. 3.

An additional problem to be considered when computing stellar models with non-grey boundary conditions is the choice of $\tau_{\mathrm{ph}}$. From this point, the integration of the atmospheric stratification supplies the values of $T_{\text {eff }}$ and $\log g$ (or luminosity and radius) for a given mass. If the atmospheric integration is consistent with the physics in the interior, and the diffusion approximation holds below $\tau_{\mathrm{ph}}$, the model location in the HR diagram should not depend on the choice of $\tau_{\mathrm{ph}}$. Since grids of atmosphere models and interiors are usually computed by different teams with different aims, quite often the physics (convection model, opacity, equation of state, etc.) used in both regions is not exactly the same. In Sect. 4 we analyze how these differences affect the evolution and structure of $1 M_{\odot}$ star.

In Sect. 5 we present our new non-grey PMS evolutionary tracks for $T_{\text {eff }} \geq 4000 \mathrm{~K}$. We have also computed two grids of "complete" FST models with metallicity larger and smaller than the solar one by a factor of 2 . The effect of different chemical compositions on HR diagram location is shown in Sect. 6.

In Sect. 7 we compare our new FST Hayashi tracks with the available data for young binary stars in star-formation regions. In fact, PMS binaries having experimentally known masses are the best test for the PMS models, since they should fit both masses and display the same age. Finally, the main conclusions of this paper are summarized in Sect. 8 .

\section{About convection in stellar modeling}

The system of equations describing stellar convection from first principles is both non-linear and non-local and its analytical or numerical solution for the general stellar case is not yet available. Even numerical simulations not resolving all the spatial scales of the flow are unaffordable for stellar evolution modeling, at least for the intermediate future, because of the excessive thermal relaxation time scales of stellar structure modeling. Non-local models such as those proposed by Xiong (1985), Canuto (1992), and Grossman (1996) are a promising and much more affordable alternative with the present computational resources. But for the time being, in stellar modeling we still stick to local models: the "standard" Mixing Length Theory (Böhm-Vitense 1958), and the "Full Spectrum of Turbulence" formalisms (Canuto \& Mazzitelli 1991; Canuto et al. 1996) are two examples.
MLT treats the heat transport with a one-eddy incompressible model with compressibility partially accounted for through the definition of a mixing length $\Lambda=\alpha H_{\mathrm{p}}$, where $H_{\mathrm{p}}$ is the local pressure scale height and $\alpha$ is a free parameter. Mimicking the spectral distribution of eddies by one "average" eddy (reliable only for high viscosity fluids) has critical consequences on the computation of MLT fluxes: Canuto (1996) showed that in the limit of highly efficient convection $(S \gg 1$, where $S$ is the convective efficiency, see e.g. CM for details) MLT underestimates the convective flux (a fact also confirmed by laboratory data, Castaing et al. 1989), and in the low efficiency limit $(S \ll 1)$ MLT overestimates the convective flux.

Computations show that inside the stars with deep convective regions $S \ll 1$ at the top of the convective envelope, just below the surface, while at the peak of the superadiabaticity $S \gg 1$. In inefficient convection, the convective temperature gradient sticks to the radiative one and begins detaching from it only when convection becomes efficient.

Historically, the choice of a mixing length $\Lambda=\alpha H_{\mathrm{p}}$ is in part a consequence of this underestimation of fluxes in MLT. Thus, the scale length $\Lambda$ was originally (Böhm \& Stückl 1967) chosen coincident with the distance $z$ from the convective boundary, consistently with the Von Karman law for incompressible fluids (Prandtl 1925), but due to the underestimation of convective flux, models with $\Lambda=z$ were not able to fit the Sun $^{3}$. Then, $\Lambda=\alpha H_{\mathrm{p}}$ grew up as standard choice, with the free parameter $\alpha$ calibrated by comparing the solar models with the actual Sun. Depending on the physical inputs the value of $\alpha$ can vary in between 1.5 and 2.2. If we use this prescription for the mixing length, at the peak of the super-adiabaticity, $\alpha H_{\mathrm{p}} \gg z$, and the fluxes, which are $\propto \Lambda^{2}$, are artificially increased.

FST (CM and CGM) models attempt to overcome the oneeddy approximation by using a turbulence model to compute the full spectrum of a turbulent convective flow. The treatment of turbulence in CM and CGM is slightly different. Nevertheless, in both cases the derived convective fluxes are quite distinct from the MLT ones. CM and CGM convective fluxes are $\sim 10$ times larger than the MLT ones for the $S \gg 1$ limit while, for the low efficiency limit $(S \ll 1)$, the CM flux is $\sim 1 / 10$ of the MLT flux, and the CGM one $\sim 0.3$ times the MLT flux. This behavior yields, in the over-adiabatic region at the top of a convection zone, steeper temperature gradients for FST than for solar-tuned MLT (and, in the FST framework, gradients steeper for CM than for CGM).

Like MLT, FST models assume the Boussinesq approximation. Following the physical argument that the Boussinesq approximation leaves no natural unit of length other than the distance to a boundary $(z)$ and the vertical stacking of the eddies due to density stratification, CM and CGM adopt respectively

\footnotetext{
${ }^{3}$ We will use the expression "fit the Sun" throughout this paper to indicate that a solar mass track of solar metallicity $Z$ reaches the present solar luminosity and radius (and thus the present solar $T_{\text {eff }}$ ) at the solar age. The fit of the solar luminosity determines the helium abundance $Y$ - or better the ratio $Z / Y$ (e.g. Turck-Chièze et al. 1988), while the fit of the radius mainly depends on the tuning of the convective model.
} 
$\Lambda_{\mathrm{CM}}=z$, and $\Lambda_{\mathrm{CGM}}=z+\alpha^{*} H_{\mathrm{p}, \mathrm{top}}{ }^{4}$. The second term in $\Lambda_{\mathrm{CGM}}$ is a fine tuning parameter that allows small adjustments, if exact stellar radii are needed, e.g., in helioseismology. Canuto et al. (1996) stress however, that the role of $\alpha^{*}$ in FST models is radically different from that of $\alpha$ in the MLT model. FST tuning affects, in fact, only layers close to the boundaries since, for inner layers, $z$ quickly grows and becomes much larger than $\alpha^{*} H_{\mathrm{p} \text {,top. }}$. On the other hand, if we wish to attribute a physical meaning to $\alpha^{*}$, the second term in $\Lambda_{\mathrm{CGM}}$ can be interpreted as an "overshooting term" representing the observed fact that convection penetrates in the stable region, and thus the scale length cannot decay to zero right at the depth where $\nabla_{\mathrm{rad}}=\nabla_{\mathrm{ad}}$ (Schwarzschild criterion of stability). In any case, $\alpha^{*}$ in these models is not properly used as an overshooting parameter but only to define the exact scale length.

The FST fluxes have also been used combined with other definitions of the mixing length. Bernkopf (1998), for instance, uses the CM version of the FST fluxes combined with a mixing length $\Lambda=\alpha H_{\mathrm{p}}$ and with $\alpha<1$.

\section{The stellar models}

The stellar models presented here were computed using the stellar evolution code ATON2.0 (Ventura et al. 1998a). Specific details concerning the computation of the pre-main sequence and deuterium burning phases are described in Mazzitelli \& Moretti (1980). We computed models for three metallicities $[\mathrm{M} / \mathrm{H}]=-0.3,0.0$, and +0.3 with an adopted helium mass fraction $Y=0.28$. Three different sets of boundary conditions were considered for solar composition: two of the new grids of ATLAS9 atmospheres from Heiter et al. (2002a), MLT ( $\alpha=0.5$ ) and CGM grids, and, for comparison, the non-grey atmosphere models by AH97. In the first case the considered masses span the interval 0.6 to $2.0 M_{\odot}$, in the second one that from 0.6 to $1.5 M_{\odot}$. For the other two chemical compositions only CGM models were computed. In addition, we computed a grey MLT evolutionary track for $1 M_{\odot}$ using for $\alpha$ the values provided by the calibration from $2 \mathrm{D}$-hydrodynamical atmosphere models (Ludwig et al. 1999).

\subsection{Equation of state and opacities}

A complete description of the equation of state (EOS) of our code is given in Montalbán et al. (2000). For the present models, the thermodynamics is from Rogers et al. (1996), for five different hydrogen abundances. At temperatures $T \geq 6000 \mathrm{~K}$ we adopt the OPAL radiative opacities $(\bar{\kappa})$ (Rogers \& Iglesias 1996, for the solar Z-distribution from Grevesse \& Noels 1993). In the high-density $(\rho)$ regions the opacities are linearly extrapolated $(\log \bar{\kappa}$ vs. $\log \rho$ ), and harmonically added to conductive opacities by Itoh \& Kohyama (1993). At lower temperatures we use the Alexander \& Ferguson's (1994) molecular opacities (plus electron conduction in full ionization) for the same $\mathrm{H} / \mathrm{He}$ ratios as in the OPAL case.

\footnotetext{
${ }^{4}$ Note that we adopt a slightly different definition of $\Lambda$ for the present computation of the interior - see Sect. 3.3.
}

\subsection{Atmospheric structure and boundary conditions}

The new grids of ATLAS9 atmospheres by Heiter et al. (2002a) introduced several improvements in comparison with previous model grids published by Kurucz $(1993,1998)$ and Castelli et al. (1997). Most of them are related to a finer grid spacing $\left(\Delta T_{\text {eff }}, \Delta \log g\right.$, but also vertical resolution) which allows a more accurate interpolation within the grids. For the convenience of the reader we recall the main characteristics of these grids:

- three different models of convection: $\operatorname{MLT}(\alpha=0.5)$, FST according to CM, and FST CGM;

- for FST models, an increase of the vertical resolution in the atmospheric integration from 72 to 288 layers ranging from $\log \tau_{\text {Ross }}=-6.875$ to $\log \tau_{\text {Ross }}=2.094$ (where $\tau_{\text {Ross }}$ is the average Rosseland optical depth);

- effective temperature range: 4000-10000 K, with $\Delta T_{\text {eff }}=200 \mathrm{~K}$;

- gravity range: $\log g$ from 2.0 to 5.0 , with $\Delta \log g=0.2$;

- chemical composition: sets for $[\mathrm{M} / \mathrm{H}]=-2.0,-1.5,-1.0$, $-0.5,-0.3,-0.2,-0.1,0.0,+0.1,+0.2,+0.3,+0.5,+1.0$ are available.

From the atmosphere models we built the tables of BCs corresponding to a fixed $\tau_{\mathrm{ph}}$. These tables contain, for each $\left(T_{\mathrm{eff}}, \log g,[\mathrm{M} / \mathrm{H}]\right)$, the following quantities: temperature $T\left(\tau_{\mathrm{ph}}\right)$, pressure $P\left(\tau_{\mathrm{ph}}\right)$, geometrical depth $z\left(\tau_{\mathrm{ph}}\right)$, geometrical depth $z\left(\tau_{0}\right)$ at the optical depth $\tau_{0}$ for which $\nabla_{\text {rad }}=\nabla_{\text {ad }}$, and finally the pressure scale height $H_{\mathrm{p}}\left(\tau_{0}\right) \equiv H_{\mathrm{p} \text {,top }}$. We built tables corresponding to $\tau_{\mathrm{ph}}=1,3,10$, and 100 in order to analyze the dependence of the results on $\tau_{\mathrm{ph}}$. We note, however, that Heiter et al. (2002a) advise to switch between model atmosphere and stellar envelope at $\tau_{\mathrm{ph}}=10$, to avoid the discrepancies due to the turbulent pressure not included in the atmosphere modeling and to reduce the effect of different opacity tables and equation of state used at both sides of $\tau_{\mathrm{ph}}$. The boundary conditions for the internal structure are determined by spline interpolation of these tables. From the initial $T_{\text {eff }}$ and $\log L$ we determine $P$ and $T$ at the last point of the internal structure $\left(\tau_{\mathrm{ph}}\right)$ and the derivative of $P$ and $T$ with respect to luminosity and radius. An iterative procedure is performed until the $P\left(\tau_{\mathrm{ph}}\right)$ and $T\left(\tau_{\mathrm{ph}}\right)$ values derived at the boundary $\tau_{\mathrm{ph}}$ from the interior or from the atmosphere models converge. In order to take into account the effect of the penetration of convection into the atmosphere, the thickness of the convection zone in the atmosphere is included in the definition of the scale length $\Lambda$ in the FST models (see the discussion of Eq. (2) below).

Some parts of the tracks for 0.6 and $0.7 M_{\odot}$ reach $T_{\text {eff }}<$ $4000 \mathrm{~K}$, falling outside the grid of ATLAS9 models. We completed the evolution by assuming as boundary conditions the values of $P$ and $T$ for the $4000 \mathrm{~K}$ grid points. The track is then artificial until $T_{\text {eff }}$ becomes again larger than $4000 \mathrm{~K}$, but this does not introduce large errors in the determination of the following evolution to the MS. We recall here that the validity of the plane parallel approximation for $\log g \geq 2$, and for the $T_{\text {eff }}$ domain of interest for our work, has already been shown by Hauschildt et al. (1999b) and Baraffe et al. (2002) for the case 


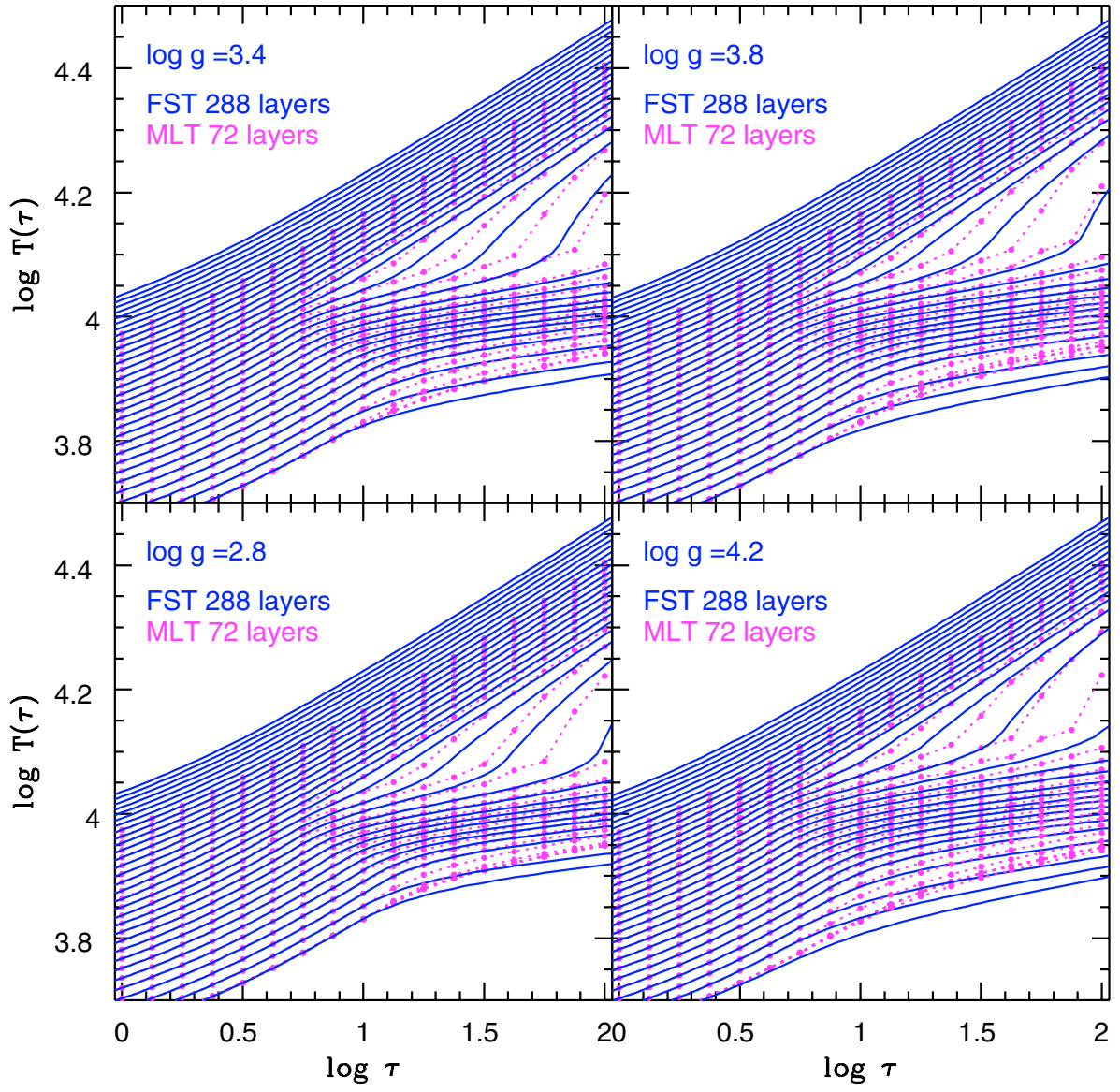

Fig. 1. $T(\tau)$ relations computed using ATLAS9, a FST treatment of convection with $\alpha^{*}=0.09$ (Heiter et al. 2002a) and integrated using 288 layers (solid lines), or a MLT one with $\alpha=0.5$ and 72 layers (dotted lines and full circles). The lower curves correspond to $T_{\text {eff }}=4000 \mathrm{~K}$ and the upper curves to $T_{\text {eff }}=10000 \mathrm{~K}$, with a step $\Delta T_{\text {eff }}=200 \mathrm{~K}$. of NextGen model atmospheres. We can hence safely assume its validity for the ATLAS9 model atmospheres as well.

Concerning the NextGen model atmospheres (AH97), their opacities include the contribution of many more molecular lines than the ATLAS9 ones, consequently, they are more adequate to study the $T_{\text {eff }}$ region where these opacities dominate (roughly, below $4000 \mathrm{~K}$ ). In AH97 models, the convection is treated with MLT and $\alpha=1$. For solar metallicity, the available models have $T_{\text {eff }}$ from 3000 to $10000 \mathrm{~K}$, and a surface gravity from $\log g=3.5$ to 6.0 , with $\Delta \log g=0.5$; the vertical resolution in the atmospheric integration is of 50 layers ranging from $\tau=10^{-7}$ to 100 (only 9 layers between $\tau=1$ and 100).

\subsubsection{The treatment of convection in the atmosphere}

The original ATLAS9 code treats the convection by using MLT with $\alpha=1.25$ (cf. Castelli et al. 1997). In addition, the code has the possibility of including a sort of "overshooting". The value $\alpha=1.25$ and the option of approximate overshooting were originally adopted to fit the intensity spectrum at the center of the solar disk and the solar irradiance. However, Castelli et al. (1997) showed that these quantities are much more sensitive to the overshooting-on mode than to the value of $\alpha$ itself (see also Heiter et al. 2002a) and that, if this treatment is included, the $\mathrm{H}_{\alpha}$ and $\mathrm{H}_{\beta}$ profiles of the solar spectrum cannot be simultaneously matched. Several papers on: $i$ ) the $T_{\text {eff }}$ determination from $\mathrm{H}_{\alpha}$ and $\mathrm{H}_{\beta}$ lines (e.g.,
Fuhrmann et al. 1993, 1994; Van't Veer-Menneret \& Megessier 1996; Van't Veer-Menneret et al. 1998); ii) the theoretical predictions of $\mathrm{H}_{\alpha}$ and $\mathrm{H}_{\beta}$ (Gardiner et al. 1999 from 1D models, and Steffen \& Ludwig 1997 from 2D numerical simulations), of $(b-y)$ and $c$ Strömgren indices (Smalley \& Kupka 1999) and of Geneva indices (Schmidt 1999), and iii) abundance determinations (Heiter et al. 1998), indicate that, even if a 1D-homogeneous model cannot explain all the spectroscopic and photometric observations, atmosphere models predicting temperature gradients closer to the radiative one (i.e., those models in which convection is less efficient than predicted by MLT-based models with $\alpha>1$ ) are in better overall agreement with observations. As pointed out, however, in the above-mentioned papers, not all the color indices over the whole $T_{\text {eff }}$ domain can be reproduced with such models either. Whereas in other cases, the quality of a fit (such as line profiles of $\mathrm{H}_{\alpha}$ ) is even independent of any sensible value of $\alpha$ (although not of other modifications to the convection treatment such as "approximate overshooting").

In the new ATLAS9-MLT grids, the convection is described as in Castelli (1996) and Castelli et al. (1997) (that is $V / A=$ $\Lambda / 6$ and $y=0.5$ ), but with $\alpha=0.5$ and without "overshooting". In the CGM model atmospheres, the convective flux is computed as in Canuto et al. (1996), but the characteristic scale length is defined as: $\Lambda=\min \left(z_{\text {top }}+\alpha^{*} H_{\mathrm{p}, \text { top }}, z_{\text {bot }}+\alpha^{*} H_{\mathrm{p}, \text { bot }}\right)$ where the index "top" and "bot" refers to top and bottom of the convective region. For most model atmospheres the difference 
with respect to the original prescription in Canuto et al. (1996) is either null or negligibly small, because the temperature gradient for convection zones which are entirely contained within the atmosphere is practically the radiative one, while, for convection zones extending below the atmosphere, the evaluation of $\Lambda$ in a pure model atmosphere has necessarily to occur near the top of the convection zone (Heiter et al. 2002a).

Since the effect of varying the fine tuning parameter $\alpha^{*}$ is felt only in a small region close to the boundary of the convection zone, changing $\alpha^{*}$ - say - by a factor two yields no difference in the solar spectrum features, so that its value cannot be calibrated from atmosphere models. Therefore, the choice of a value of $\alpha^{*}=0.09$ was based on the solar calibration performed by Canuto et al. (1996), but it has to be pointed out that this value was determined using grey BCs (Henyey et al. 1965). As shown in Heiter et al. (2002a) the temperature structure in the deep atmosphere is strongly dependent on the convection model (Fig. 1). Because of the features of convective fluxes described in Sect. 2, the convection modeled with MLT $(\alpha=0.5)$ is very inefficient in the whole atmosphere, while the FST models, which are similarly inefficient at the outer boundary, rapidly begin to be very efficient in the deeper layers of solar-like convection zones. In Fig. 2 we plot the overadiabaticity and the jump of specific entropy corresponding to either MLT or FST atmosphere models with two different values of $T_{\text {eff }}$ and $\log g$. The stellar spectra are not sensitive to the deep atmosphere gradients, and from the point of view of atmosphere models, as long as one uses a low value of $\alpha$, MLT and FST are essentially equivalent. The BCs provided by both atmosphere models can become, however, very different as the optical depth where the BCs are taken increases.

\subsubsection{Resolution}

Schmidt (1999) showed that a scale length prescription as used in CM or CGM together with a low vertical resolution could result in "jumps" in the grids of $\max \left(F_{\text {conv }} / F_{\text {tot }}\right)$ as function of $T_{\text {eff }} / \log g$. That is due to the fact that the convective zone expands in total size and retreats from the upper/mid photosphere when going from high- $T_{\text {eff }} /$ low- $g$ to low- $T_{\text {eff }} /$ high $-g$. A change of the vertical extension of the convective zone even of a single layer can have a significant impact on the value of $\Lambda(z)$, and hence on $F_{\text {conv }}\left(\sim \Lambda^{8}\right.$ in the low efficiency regime, see Gough \& Weiss 1976 and references therein). Another aspect of the same problem was detected in computing FST stellar models with 72-layers atmosphere models as BCs. The thickness of the convection zone in the atmosphere $\Lambda_{\mathrm{atm}}=z\left(F_{\text {conv }}=0\right)-z\left(\tau=\tau_{\mathrm{ph}}\right)$ as a function of $\log g$ and $T_{\text {eff }}$ was far from being smooth, in particular in the low- $T_{\text {eff }} /$ low- $g$ region of the HR diagram, where convection quickly extends into the higher atmosphere layers due to the contribution of $\mathrm{H}^{-}$and $\mathrm{H}_{2}$ to the opacity. So, the resulting evolutionary paths in the HR diagram showed oscillations which disappear when new models with 288 layers are used.

The comparison between the $T(\tau)$ 's relations for CGM-atmosphere models integrated with 72 and with 288 layers (see Fig. 1 in Montalbán et al. 2002) illustrates a result

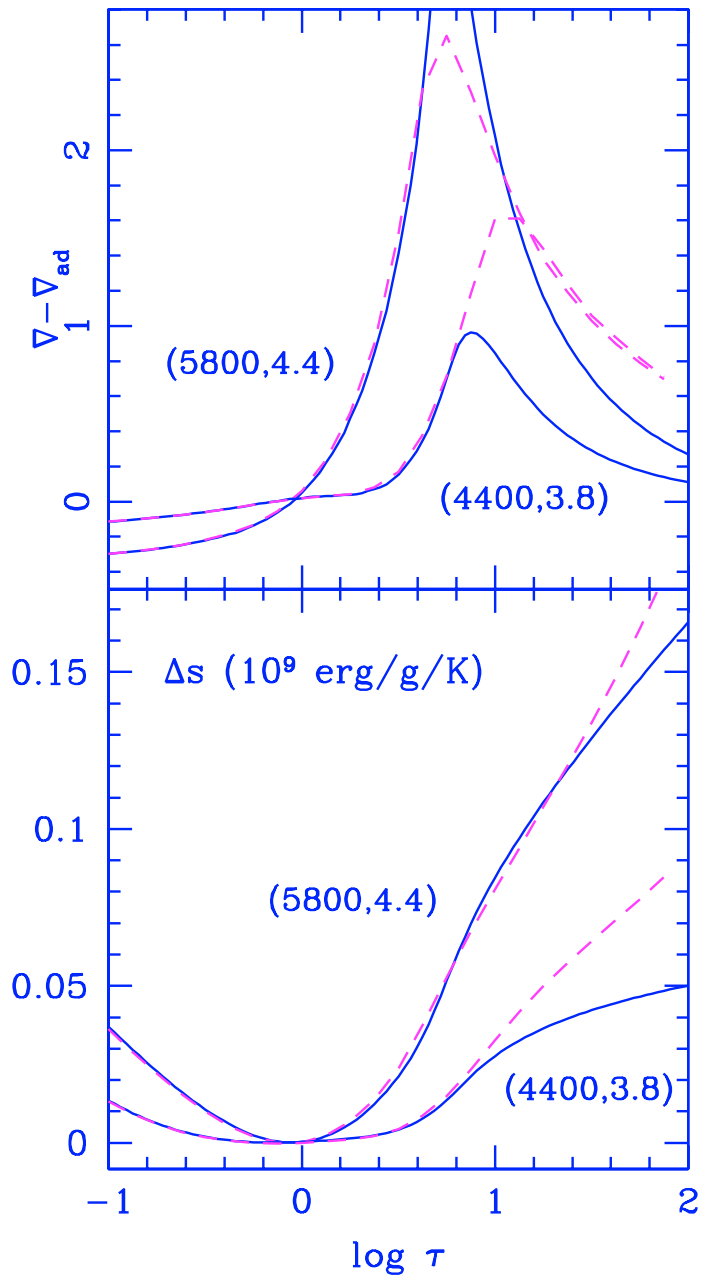

Fig. 2. Upper panel: over-adiabaticity as a function of the optical depth in the atmosphere for two pairs of $\left(T_{\text {eff }}, \log g\right)$ corresponding to typical values for a $1 M_{\odot}$ star during MS and PMS. Dashed lines correspond to MLT ATLAS9 $\left(\alpha_{\mathrm{atm}}=0.5\right)$ atmosphere models, and solid lines to CGM FST ones. Lower panel: entropy jump for the same atmospheres as in upper panel.

which from a different point of view was also discussed by Heiter et al. (2002a): in the case of ionization regions it is possible to correctly follow the rapid increase of temperature only if the number of layers is large enough. The same figure also points out a large uncertainty introduced for $\mathrm{F}$ and $\mathrm{G}$ stars (depending on their surface gravity), when the boundary condition is taken at optical depths $\tau_{\text {ph }}$ larger than 20 , because of the onset of (nearly) adiabatic convection in the envelope of cool stars compared to the (almost) radiative temperature gradient of hotter stars.

Atmosphere models are usually built to generate synthetic spectra to be compared with the observations. As a consequence, the number of mesh points per unit optical depth must be kept large in the region where the continuum and the lines are formed, that is at low optical depth, while few points are computed in between $\tau=10$ and $\tau=100$, though this is the recommended region (Morel et al. 1994) to adopt the values of temperature and pressure as BCs for the internal structure computation. The differences between $T(\tau)$ 's relations corresponding to 72-CGM and 288-CGM atmosphere models show 
that low resolution models could induce non-negligible errors in the BCs, especially if $\tau_{\mathrm{ph}}=100$ is chosen as match point between the atmosphere and the interior.

\subsection{Convection modeling in the interior}

For the interior as well, models are computed either by MLT or FST. If we want to build complete stellar models using the MLT treatment of convection and fit the Sun, we should use $\alpha$-values in the interior $\left(\alpha_{\text {int }}\right)$ much larger than that one in the atmosphere $\left(\alpha_{\text {atm }}=0.5\right)$ (see Sect. 4$)$.

The MLT used in the interior strictly follows the formulation of Cox \& Giuli (1968), who chose the $V / A$ relation in order to obtain numerical agreement with the results of Böhm-Vitense (1958). In the atmosphere, as mentioned above, the MLT formulation follows Castelli (1996). There, the choice of $y$ and $V / A$ parameters is such that for the same $\alpha$-value, the efficiencies of convection in Cox \& Giuli formulation $\left(\Gamma_{\mathrm{CG}}\right)$ and in Castelli $\left(\Gamma_{\mathrm{C}}\right)$ hold the relation $\Gamma_{\mathrm{CG}}=2 / 3 \Gamma_{\mathrm{C}}$. This difference is much smaller than that introduced in the efficiency of convection by varying the $\alpha$-value from -say- 1.7 to 0.5 .

The FST model of convection adopts the CGM fluxes, and the scale length at the radial coordinate $r$ is the harmonic average between $z_{\text {top }}$ (distance to the top of convection increased by $\alpha^{*} H_{\mathrm{p} \text {,top }}$ ) and $z_{\text {bot }}$ (distance to the bottom of convection increased by $\alpha^{*} H_{\mathrm{p}, \mathrm{bot}}$ ), namely

$\Lambda=z_{\text {top }} z_{\text {bot }} /\left(z_{\text {top }}+z_{\text {bot }}\right)$.

This choice ensures that, close to the boundaries, $z \sim z_{\text {top }}$ or $z \sim$ $z_{\text {bot }}$, and, far from boundaries, $\Lambda \sim H_{\mathrm{p}}$, as it should be expected (for details see Ventura et al. 1998a). For the models in which convection penetrates in the atmosphere, the quantity $z_{\text {top }}$ has to include the distance from the bottom of the atmosphere $\left(z\left(\tau_{\mathrm{ph}}\right)\right)$ to the top of convection in the atmosphere $\left(z\left(\tau_{0}\right)\right)$, and $H_{\mathrm{p} \text {,top }}$ is also evaluated at $z\left(\tau_{0}\right)$ in the atmosphere. So,

$z_{\text {top }}(r)=\left[z\left(\tau_{0}\right)-z\left(\tau_{\mathrm{ph}}\right)\right]+\left(R_{\max }-r\right)+\alpha^{*} H_{\mathrm{p}}\left(\tau_{0}\right)$,

where $r$ is the radius of the layer and $R_{\max }$ is the radius of the last point computed by the integration from the interior, that is the radius of the boundary point $\left(\tau_{\mathrm{ph}}\right)$.

The fine tuning parameter $\alpha^{*}$ for the computation of the interior can also be changed in order to fit the Sun. We will call $\alpha_{\text {int }}^{*}$ the parameter used for computation in the interior, in contrast with the fixed value $\alpha_{\mathrm{atm}}^{*}=0.09$ adopted in the computation of the grid of atmosphere models ${ }^{5}$.

\section{Effect of $\tau_{\mathrm{ph}}$ on the evolutionary track location}

Since it is not reasonable by now to include a full atmosphere calculation into the computation of a whole stellar model, one separates the internal structure calculation, where the diffusion approximation is used, from the outer part. The calculation of

\footnotetext{
${ }^{5}$ We will approximate this value $\alpha_{\text {atm }}^{*}=0.09$ with 0.1 in Table 1 and in the text. This is due to the fact that we have used $\alpha_{\text {int }}^{*}=0.1$ in the interior computations. For any purpose, 0.09 and 0.1 provide practically the same results.
}

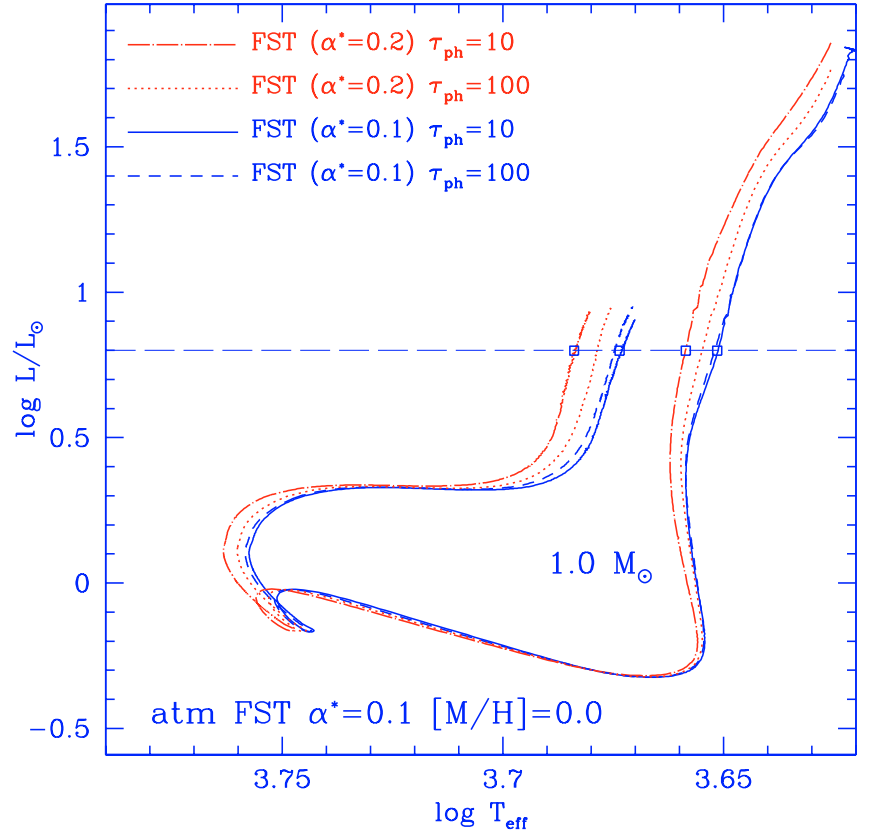

Fig. 3. FST evolutionary tracks for $1 M_{\odot}$ for two different choices of the tuning parameter $\alpha^{*}$ and two different choices of the subphotospheric boundary, $\tau_{\mathrm{ph}}=10$ and 100 . The horizontal line refers to the models in Fig. 4.

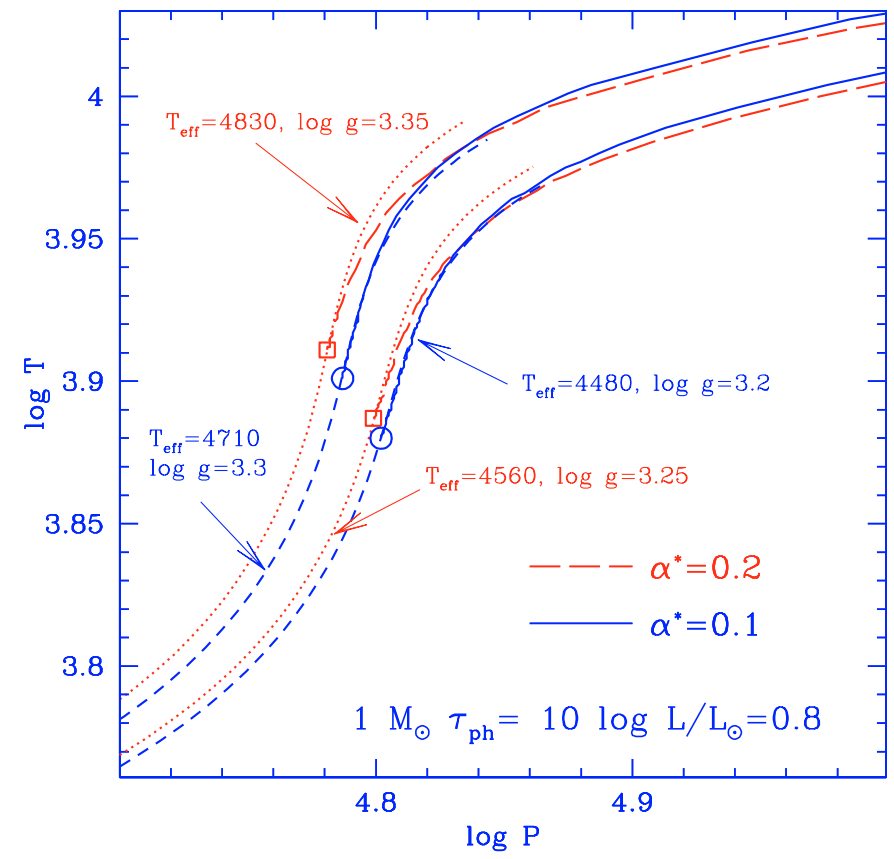

Fig. 4. Match between FST internal structure $\left(\alpha^{*}=0.1\right.$, solid line; $\alpha^{*}=0.2$, long-dashed line) and the corresponding FST atmospheres (short-dashed and dotted lines) for two different evolutionary phases of $1 M_{\odot}$. The models correspond to the square points in Fig. 3 .

the atmosphere is replaced by the values of the relevant physical quantities at the optical depth $\tau_{\mathrm{ph}}$. This $\tau_{\mathrm{ph}}$ must be large enough for the diffusion approximation for $\tau>\tau_{\mathrm{ph}}$ to be valid, and small enough for the approximations adopted for the description of the physics in the atmosphere to hold. Morel et al. (1994) proposed $\tau_{\mathrm{ph}} \geq 10$, depending on $T_{\mathrm{eff}}$ and $\log g$ of the model. 
If the calculations of the interior and the atmosphere were physically consistent, the stellar models computed with different choices of $\tau_{\mathrm{ph}}$ should be equivalent. In practice, the physical inputs such as chemical composition, opacity tables, equation of state, and convection model used in the atmosphere are usually not exactly the same as those used in the interior.

The atmosphere models by Heiter et al. (2002a) adopt the opacity and the equation of state from Kurucz $(1993,1998)$, while our stellar evolution code considers the micro-physics inputs described in Sect. 3.1. Figure 3 shows, however, that, if the same convection model (CGM, $\alpha^{*}=0.1$ ) is used in the atmosphere and in the interior, the HR diagram location of $1 M_{\odot}$ evolutionary tracks is almost independent of the choice of $\tau_{\mathrm{ph}}$. Figure 4 shows the structures $(\log T$ vs. $\log P$ ) of the interiors and the corresponding atmospheres of $1 M_{\odot}$ models at different evolutionary phases (PMS and Post-MS models marked in Fig. 3 by square points). We see that, in spite of differences in the opacity tables and in the equations of state, the atmosphere structures between $\tau=10$ and 100 almost overlap the values ( $\log T$ vs. $\log P$ ) obtained for the outer layers by integrating the internal structure. The differences introduced in $T_{\text {eff }}$ due to the different choices $\tau_{\mathrm{ph}}=10$ or 100 are smaller than $0.5 \%$ along the evolutionary track of $1 M_{\odot}$ with solar metallicity. This is not true if we consider smaller values of $\tau_{\mathrm{ph}}$ : for $\tau_{\mathrm{ph}}=1$ or $\tau_{\mathrm{ph}}=3$ the differences in temperature gradients between the interior and the atmosphere are larger, because the diffusion approximation of radiative energy transport used in the internal computation is not valid. The temperature gradient computed in the stellar structure code begins to deviate from the atmospheric one at an optical depth lower than $\sim 7$, but the exact point depends on $T_{\text {eff }}$ and on $\log g$. Consequently, the details in the equation of state and opacities are of much less a concern for matching atmosphere and interior models than the region of validity of the diffusion approximation and the modeling of convection, to which we now turn our main attention.

\subsection{Solar calibrations}

It is generally accepted that any stellar modeling must be able to fit the Sun. Two parameters control the Sun's location in the HR diagram: the helium content which is determined by the solar luminosity, and the jump of specific entropy $(\Delta s)$ between the photosphere and the adiabatic convection region (e.g., Christensen-Dalsgaard 1997) which determines the stellar radius.

$\Delta s=\int_{\ln p_{0}}^{\ln p_{\mathrm{ad}}} c_{\mathrm{p}}\left(\nabla-\nabla_{\mathrm{ad}}\right) \mathrm{d} \ln p$,

where $p_{0}$ refers to the pressure at a given point in the photosphere, and $p_{\text {ad }}$ is the pressure at the point in the convective zone where $\left(\nabla-\nabla_{\mathrm{ad}}\right) \ll 1$.

In MLT, $\left(\nabla-\nabla_{\mathrm{ad}}\right)$ is directly related to the $\alpha$ parameter, and thus varying $\alpha$ allows the fit of solar radius. Hence, depending on the physical inputs, grey models provide values of $\alpha$ between 1.5 and 2.2 (Ventura et al. 1998a). Using the CGM model of convective flux and scale height, only a small variation of $\left(\nabla-\nabla_{\mathrm{ad}}\right)$ can be obtained by varying the tuning parameter $\alpha^{*}$ and for a solar grey model $\alpha^{*} \simeq 0.1$ (CGM, Ventura et al. 1998a) is required. For the present models, using the relations $T(\tau)$ provided by the grids of non-grey atmospheres, the value of $\alpha$ (and $\alpha^{*}$ in the FST, but see later) required to compute the convective envelope fitting the Sun depends also on the choice of $\tau_{\mathrm{ph}}$. Equation (3) can be divided in two terms, $\Delta s_{\mathrm{atm}}\left(\tau_{\mathrm{ph}}\right)$ (jump of specific entropy from the surface to $\left.\tau_{\mathrm{ph}}\right)$ and $\Delta s_{\text {int }}\left(\tau_{\mathrm{ph}}\right)$ (s jump between $\tau_{\mathrm{ph}}$ and a layer deep enough where $\left.\left(\nabla-\nabla_{\mathrm{ad}}\right) \sim 0\right)$. Thus, the contribution of the model atmosphere to the entropy jump $\Delta s$ depends crucially on $\tau_{\mathrm{ph}}$ and for any chosen $\tau_{\mathrm{ph}}, \Delta s_{\mathrm{atm}}\left(\tau_{\mathrm{ph}}\right)$ must be smaller than $\Delta s$ for a solar model to fit the Sun.

We have considered several combinations of convection treatment in the interior and in the atmosphere, and several choices of $\tau_{\mathrm{ph}}$. Table 1 lists the different sets of models computed for the solar composition. The set of parameters fitting the Sun are indicated with a numerical super-script. The value of this index corresponds to the labels in Figs. 5 and 6.

For CGM models, with the non-grey BCs the choice of $\alpha^{*}=0.1$ as derived by the solar calibration with grey BCs provides a Sun $\sim 85 \mathrm{~K}$ cooler than expected. To fit the Sun requires, hence, a larger $\alpha^{*}$ value in the computation of the interior: viz. $\alpha^{*} \sim 0.2$ if $\tau_{\mathrm{ph}} \leq 10$ (Fig. 4). Models computed with consistent $\alpha^{*}$ in the interior and in the atmosphere would have an intermediate value of $\alpha^{*}$. As shown in Fig. 4, an increase of $\alpha^{*}$ by a factor 2 implies a lower temperature gradient in the layers deeper than $\tau_{\mathrm{ph}}=10$ and hence a higher $T_{\text {eff }}$. The term $\alpha^{*} H_{\mathrm{p}}\left(\tau_{0}\right)$, however, affects only the scale length close to the boundary of the convective zone. Since the efficiency of FST convection rapidly increases downwards the convective zone, the atmosphere at $\tau=100$ is quasi-adiabatic (see Fig. 2) and hence even a value of $\alpha^{*}$ larger, but restricted to the interior, cannot increase the entropy in the adiabatic region. This explains what is meant by saying that the FST model with the scale length as in Eqs. (1)-(2), or equivalently with the original suggestion from Canuto et al. (1996), is less parametric than standard MLT: if an FST-based solar model fails to fit the Sun, there is less possibility of achieving anyway a match by adjusting $\alpha^{*}$, as this parameter only provides some fine tuning ${ }^{6}$.

Concerning the MLT calibration, Fig. 2 shows an atmosphere with $T_{\text {eff }}$ and $\log g$ close to the solar values. The over-adiabaticity is quite high up to $\tau=100$ and therefore $\Delta s_{\mathrm{atm}}\left(\tau_{\mathrm{ph}}=100\right) \gg \Delta s_{\mathrm{atm}}\left(\tau_{\mathrm{ph}}=10\right)$. To keep $\Delta s$ in the range required to fit the solar radius, the contribution of the term $\Delta s_{\text {int }}\left(\tau_{\text {ph }}\right)$ is controlled by increasing $\alpha_{\text {int }} \operatorname{since}\left(\nabla-\nabla_{\text {ad }}\right) \sim$ $\alpha^{-4 / 3}$ in the high efficiency limit. This explains the very different values of $\alpha_{\text {int }}$ required with different $\tau_{\mathrm{ph}}$ 's ( $\alpha_{\mathrm{int}}=2.3$ for $\tau_{\mathrm{ph}}=10$, and $\alpha_{\mathrm{int}}=6.3$ for $\tau_{\mathrm{ph}}=100$ ). Obviously, this results in a kink at the connection point, inducing discontinuities in the derivatives of the temperature gradient and in the over-adiabaticity (cf. Fig. 6). A temperature structure similar to that obtained with FST could be obtained with MLT and a variable length scale, as in Schlattl et al. (1997). These authors found that between $\tau\left(T=T_{\text {eff }}\right)$ and $\tau=20$ a MLT 1-D

\footnotetext{
${ }^{6}$ In principle, the FST convective fluxes could also be used with a scale length $\alpha H_{\mathrm{p}}$, but no grids of model atmospheres are available for this case.
} 
Table 1. Solar-composition computed models.

\begin{tabular}{ll|lc|l}
\hline \hline Atmosphere & & Interior & & $\tau_{\mathrm{ph}}$ \\
\hline ATLAS9 MLT & $\alpha=0.5$ & MLT & $\alpha=2.3$ & $10^{(3)}$ \\
ATLAS9 MLT & $\alpha=0.5$ & MLT & $\alpha=6.3$ & $100^{(2)}$ \\
ATLAS9 MLT & $\alpha=0.5$ & MLT & $\alpha=1.75$ & $1^{(4)}$ \\
ATLAS9 MLT & $\alpha=0.5$ & MLT & $\alpha=1.85$ & 3 \\
ATLAS9 FST & $\alpha^{*}=0.1$ & FST & $\alpha^{*}=0.1$ & $1,3,10,100$ \\
ATLAS9 FST & $\alpha^{*}=0.1$ & FST & $\alpha^{*}=0.2$ & $1,3,10^{(1)}, 100$ \\
AH97 MLT & $\alpha=1.0$ & MLT & $\alpha=1.0$ & $3,10,100$ \\
AH97 MLT & $\alpha=1.0$ & MLT & $\alpha=1.9$ & $3,10,100^{(5)}$ \\
\hline
\end{tabular}

atmosphere, computed with $\alpha=0.5$, agrees very well with the 2D-hydrodynamical model from Freytag et al. (1996). Hence they decided to use a model atmosphere computed with $\alpha=0.5$ down to $\tau=20$ and introduced a mixing-length parameter $\alpha=\alpha(\tau)$ whose dependence on $\tau$ was chosen to model the temperature-pressure stratification of the 2D-model, and the parameters to fit the solar values of $T_{\text {eff }}$ and luminosity (see their Fig. 1).

Clearly, such procedures can be applied to the solar case, but how to extrapolate them to other regions of the HR diagram? Although these MLT-based models are built in such a way that the atmospheric convection prescription fits the solar Balmer lines better than the standard models with large $\alpha$, and the internal structure integration fits the present solar radius, we have to warn about the poor physical meaning of extending this procedure to structures different from the Sun. To fit the Sun, the low $\alpha=0.5$ needed in the atmosphere is compensated by the larger value adopted in the interior, but the effect on the evolutionary track of a different $\alpha$-value below $\tau_{\mathrm{ph}}$, is not the same over the whole HR diagram. As shown in Fig. 5, very different PMS evolutionary tracks (by $T_{\text {eff }}$ and shape) are obtained with parameter sets all fitting the HRD location of the Sun. We note that the PMS track of the $1 M_{\odot}$ model, obtained integrating the interior up to $\tau_{\mathrm{ph}}=1$, is, as expected, quite close to the MLT grey model. Moreover the coolest low-PMS tracks are obtained when the integration of the atmosphere (with low efficiency convection, $\alpha_{\mathrm{atm}}=0.5$, or 1.0) goes down to $\tau_{\mathrm{ph}}=100$. But, which are the good PMS tracks: those obtained with $\tau_{\mathrm{ph}}=10$, or with $\tau_{\mathrm{ph}}=100$ ? The convection properties of stellar layers at $\tau_{\mathrm{ph}}$ are not the same all over the HR diagram. The fraction of the over-adiabatic region above $\tau_{\mathrm{ph}}$ depends on $T_{\mathrm{eff}}$ and $\log g$. So, there is no physical reason in choosing an efficiency of convection below $\tau_{\mathrm{ph}}$ and another above that point. The only justification for doing so could be the match of a larger set of observed data, but this procedure would require calibration standards throughout the HR diagram. On the other hand, the procedure of mixing very different treatments of convection, based on solar calibration, finally results in physical inconsistencies, introduces a large dispersion $(\sim 200 \mathrm{~K})$ in the low gravity region of the HR diagram (see Fig. 5), and reduces even more the predictive power of MLT based stellar modeling.

Ludwig et al. (1999) proposed a calibration of $\alpha\left(T_{\mathrm{eff}}, \log g\right)$ providing the same specific entropy jump in a grey atmosphere

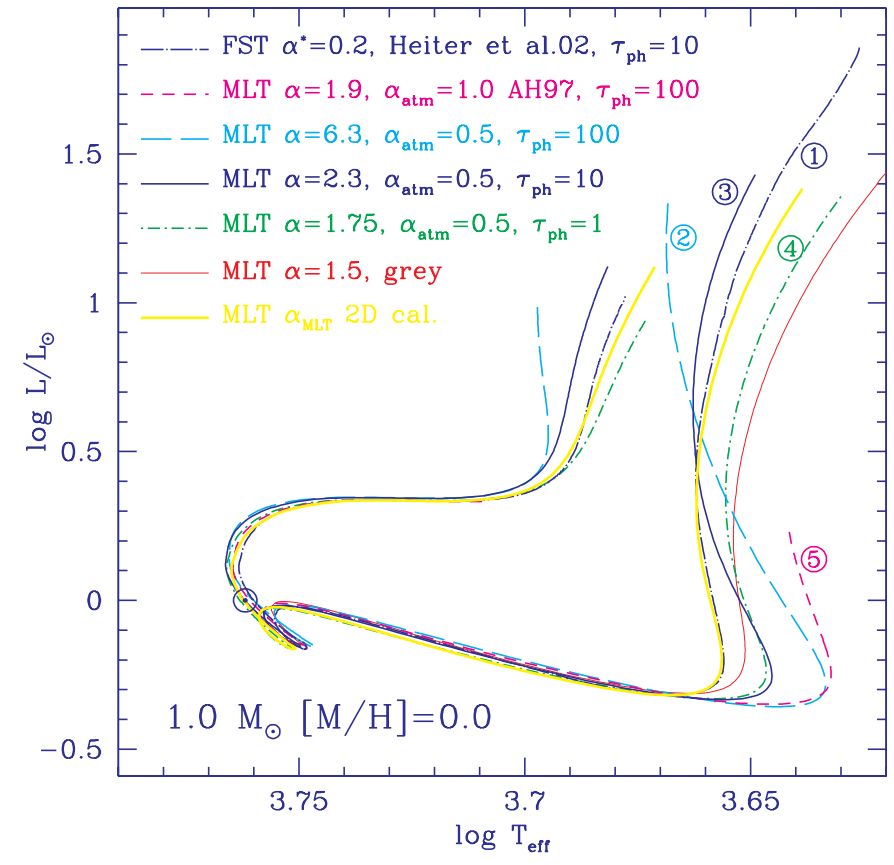

Fig. 5. Several evolutionary tracks for $1 M_{\odot}$, solar composition, and MLT treatment of convection in the interior and in the atmosphere. Different values of $\alpha$ for each choice of $\tau_{\mathrm{ph}}$ are required to fit the Sun with MLT atmospheres from Heiter et al. (2002a). Besides, a grey MLT model with the same opacity and EOS, and the new FST track with $\alpha^{*}=0.2$ in the interior and FST atmospheres by Heiter et al. (2002a) are plotted. The thick line corresponds to a grey MLT model computed with the $\alpha$ value given by the 2D numerical simulations calibration of Ludwig et al. (1999).

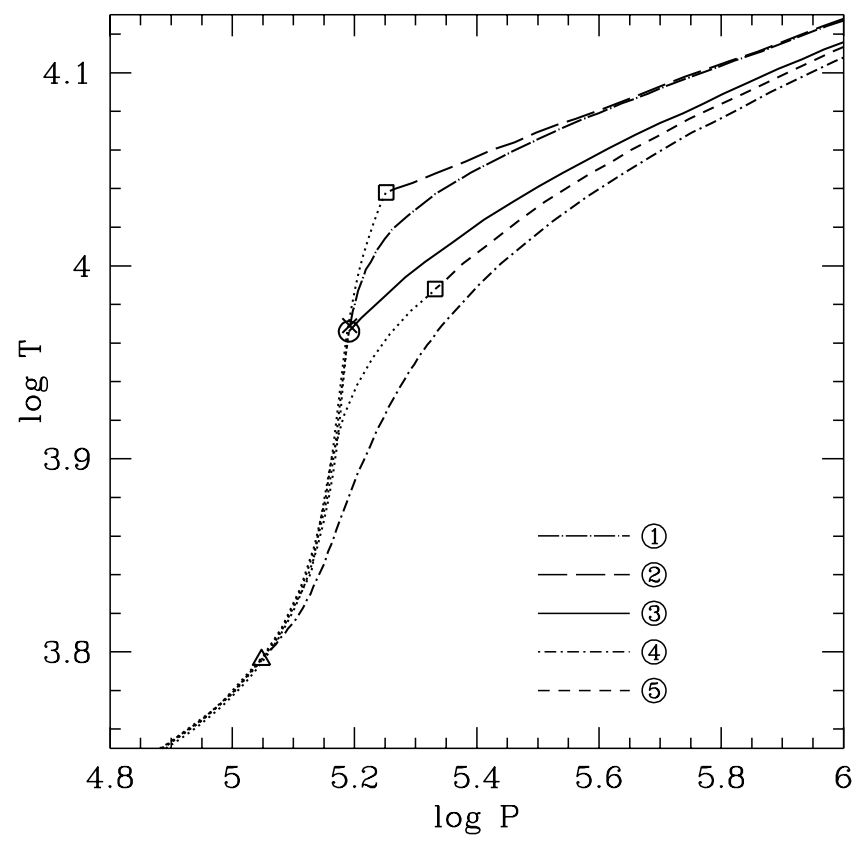

Fig. 6. Stellar structure, interior and atmosphere for the solar model corresponding to the labeled evolutionary tracks as obtained with the evolutionary tracks plotted in Fig. 5. MLT models: atmosphere model with $\alpha_{\mathrm{atm}}=0.5, \tau_{\mathrm{ph}}=100$ (long-dashed line), $\tau_{\mathrm{ph}}=10$ (solid line), $\tau_{\mathrm{ph}}=1$ (short-dashed-dotted line); atmosphere model from AH97, $\alpha_{\text {atm }}=1, \tau_{\text {ph }}=100$ (short-dashed line); FST model (long-dasheddotted line). The circle, squares, triangle and cross indicate the match point between interior and atmosphere. 
as their 2-D numerical simulations, and therefore, the same stellar radius (although this does not imply the model will also have a realistic sub-photospheric stellar structure). The thick line in Fig. 5 was computed with the same EOS and opacity tables, but adopting a grey atmosphere and MLT with the $\alpha\left(T_{\text {eff }}, \log g\right)$ 2D-based calibration. We see that for $\log L / L_{\odot}<0.75$ this track almost overlaps that obtained by non-grey FST modeling.

Figure 6 shows how different the sub-atmospheric layers of the "Sun's" can be, as obtained from five sets of parameters fitting the solar $T_{\text {eff }}$ and radius. Thanks to helioseismology it is possible to get information about the interior of the Sun, and several authors (Baturin \& Miranova 1995; Monteiro et al. 1995; Heiter et al. 2002b from non-grey MLT and FST models) have concluded that FST solar models yield p-mode frequencies which are in better agreement with the helioseismological data. Schlattl et al. (1997) as well, with their spatially varying mixing length parameter, obtained clear improvements with respect to previous computations. It has to be noticed that even if the abrupt change of slope in the temperature profiles \#2 and \#3 of Fig. 6 is adequately smoothed, the frequencies resulting from both kinds of sub-atmospheric structures (FST and MLT with $\tau_{\mathrm{ph}}=10$ ) will be different and their differences can hence be probed by helioseismology.

The matching of model atmospheres on top of interior structure calculations raises the question if it is possible to put a constraint on the optical depth where convection becomes efficient and if low values of $\alpha$ in an MLT treatment can be excluded. The case of stars with shallow - in terms of $H_{\mathrm{p}}-$ surface convection zones, such as A-type main sequence stars, can be described in terms of inefficient convection throughout the convective zone, from an observational point of view (Smalley \& Kupka 1997; Heiter et al. 2002a; Smalley et al. 2002), from the results of non-local convection models without a mixing length, and from numerical simulations (see the discussion in Kupka \& Montgomery 2002; Freytag 1995). Unfortunately, for stars with deep envelope convective zones the interpretation of the observational data remains ambiguous (cf. also Castelli et al. 1997; Heiter et al. 2002a) and the comparison with numerical simulations provides no simple alternative, i.e. a parameterization of temperature and pressure as a function of depth which could simultaneously fit both the stellar radii and the spectroscopic and photometric data. Obviously, the calibration problem becomes even worse for the case of evolutionary tracks running through extended regions of the HR diagram. In comparison with MLT, the FST based models offer the advantage of agreeing with more observational data without the need of drastically changing the scale length at some ill-defined matching depth, which, in the end, introduces at least another free parameter. Nevertheless, the discrepancies of these models with observed color photometry for the Sun (Smalley \& Kupka 1997 and references therein) and with temperature gradients as obtained from various 3D large eddy simulations of solar granulation call for urgent further improvement in convection modeling.

\section{Convection treatment and PMS location}

From Fig. 5 it is clear that HR diagram location of the Hayashi tracks is strongly model-dependent, and the models with different convection treatment - even selecting only those models fitting the Sun - provide tracks of very different shapes and $T_{\text {eff }}$ ranges (see also the first discussion given by D'Antona $\&$ Mazzitelli 1994). In this section we show the origin of differences and similarities among available PMS evolutionary tracks, often used in comparing and interpreting observational data.

\subsection{FST based models}

In this section we analyze the effect of different formulations of the FST model (different $\alpha^{*}$, plus the grey model) on the PMS evolution. As seen in the previous section, the solar calibration with non-grey BCs requires a tuning of the parameter $\alpha^{*}$ larger by a factor of two with respect to that used for the atmospheric grids. No feature from spectra and colors can constrain this parameter, but the deep structure of the atmosphere will be slightly different. The new value $\alpha^{*}=0.2$ required when $\tau_{\mathrm{ph}} \leq 10$ is anyway within the limits given for stellar "overshooting" (Maeder \& Meynet 1991). In principle, an inconsistency is introduced in this way between the treatment of convection in outer and internal layers. The effects of this inconsistency on the HR diagram are small, but different for each $\left(T_{\text {eff }}, \log g\right)$. For $T_{\text {eff }}<4700 \mathrm{~K}$ the atmospheric convection begins at increasingly shallower layers and becomes almost adiabatic deep in the FST atmosphere (see curve corresponding to $T_{\text {eff }}=4400 \mathrm{~K}$ and $\log g=3.8$ in Fig. 2). This explains the small effect of different $\alpha^{*}$ on the PMS tracks location in the right side of the HRD compared with the effect on the PMS tracks corresponding to the highest masses considered.

Figure 7 shows the evolutionary tracks for masses from 0.6 to $2.0 M_{\odot}$ obtained using: $i$ ) exactly the same treatment of convection in the atmosphere and inside the star (dotted lines), i.e. CGM fluxes and scale length with $\alpha^{*}=0.1$; and ii) a slightly different scale length with $\alpha_{\mathrm{atm}}^{*}=0.1$, and $\alpha^{*}=0.2$ below $\tau_{\mathrm{ph}}=10$. The effect on $T_{\text {eff }}$ of changing $\alpha^{*}$ by a factor 2 will depend on the quantity $a=\left(\Lambda_{\text {atm }}+\Lambda_{\text {int }}\right) /\left(\alpha^{*} H_{\mathrm{p}}\right)$ (where $\Lambda_{\text {atm }}$ is the extent of the convection zone for $\tau<\tau_{\mathrm{ph}}$ and $\Lambda_{\text {int }}$ is the distance to $\tau_{\mathrm{ph}}$ ). If $a$ is much larger than one, the variation of $T_{\mathrm{eff}}$ will be negligible as the convective flux and the temperature gradients will not change. On the contrary, if it is of the order of one or smaller, the effect on the temperature gradients for the last points of the structure will be significant and as the gradients decrease, the $T_{\text {eff }}$ 's increase. The differences of $T_{\text {eff }}$, at constant luminosity, induced by the tuning of $\alpha^{*}$ go from $\sim 30 \mathrm{~K}$ at the lowest masses, to $\sim 80 \mathrm{~K}$ for $1.5 M_{\odot}$, and $\sim 120 \mathrm{~K}$ for $2.0 M_{\odot}$. This implies that, at given $L$ and $M$, the maximum uncertainty introduced in $T_{\text {eff }}$ is $\sim 2 \%$ ( $<1 \%$ for $\left.1 M_{\odot}\right)$; and, at a given $L$ and $T_{\text {eff }}$ the maximum uncertainty in mass is $\sim 17 \%$.

Figure 8 shows the effect of non-grey BCs on the location of the Hayashi tracks. DM97, 98 models were computed with the CGM formulation of convection in the interior and with grey BCs. The authors approached the problem of convection penetrating in the atmospheres of low-gravity/low-temperature 


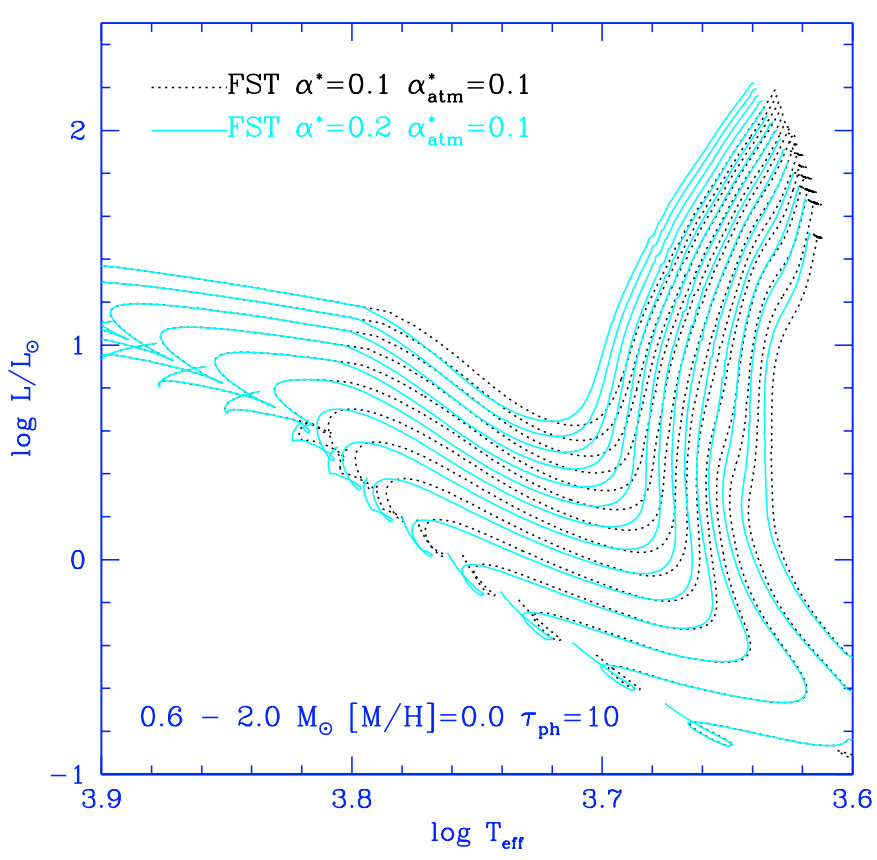

Fig. 7. FST evolutionary tracks with ATLAS9-FST atmospheres with $\alpha^{*}=0.1$.

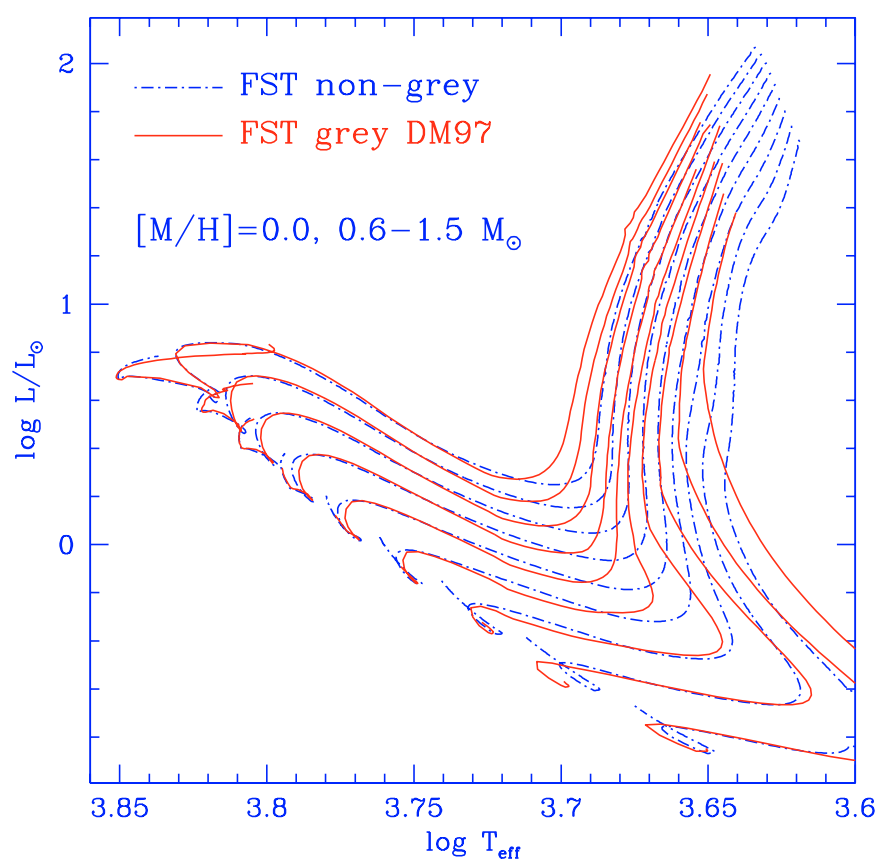

Fig. 8. Comparison between FST grey models (DM97), and "complete" FST models with the new ATLAS9 atmosphere models.

models, integrating the interior until the top of the convective region. The present "complete" (and more physically coherent) FST models are much cooler than the grey ones, $\sim 270 \mathrm{~K}$ for the lowest computed masses.

\subsection{Effect of different MLT prescriptions}

We computed as well two grids of stellar models with the same micro-physics in the interior, but using NextGen atmosphere models (AH97) as BCs. NextGen includes a large number of

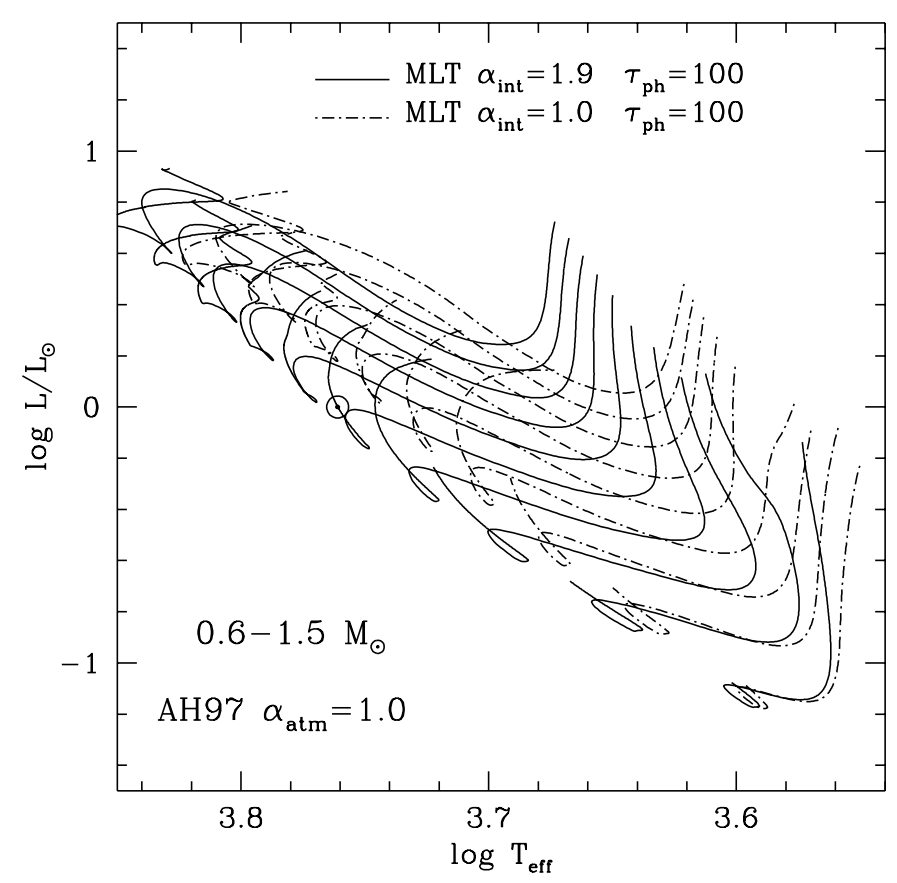

Fig. 9. MLT PMS tracks obtained using as BCs the atmosphere models by AH97 ( $\alpha=1$ ), with two different values of $\alpha$ in the computation of the sub-atmospheric convection and with the match point as in BCAH98, $\tau_{\mathrm{ph}}=100$.

molecular lines which, being the main source of opacity for low-temperature models, must have a significant role in the onset of convection. These models use MLT with $\alpha=1.0$ and provide super-adiabatic gradients smaller than those from the new ATLAS9 models. As a consequence, adiabaticity is reached at lower depths than in ATLAS9 (MLT $\alpha=0.5$ ) models and there is a smaller difference in choosing either $\tau_{\mathrm{ph}}=10$ or $\tau_{\mathrm{ph}}=100$.

Anyway, the value $\alpha_{\text {int }}=1$ is too small to fit the solar radius, for which $\alpha_{\text {int }}=1.9$ is required. Figure 9 shows the tracks computed with NextGen atmospheres $\left(\alpha_{\mathrm{atm}}=1.0\right)$ down to $\tau_{\mathrm{ph}}=100$, and with $\alpha_{\text {int }}=1.0$ (dash-dotted curve) and $\alpha_{\text {int }}=$ 1.9 (solid line) for all layers below. These curves are equivalent to those published by Baraffe et al. (1998, BCAH98). We point out that there is a large difference in $T_{\text {eff }}(\sim 380 \mathrm{~K}$ in MS and $440 \mathrm{~K}$ in PMS for a $1 M_{\odot}$ model) and that the shape of the Hayashi tracks as well changes with the $\alpha$-value adopted in the interior. The NextGen tracks with the parameter fitting the Sun $\left(\alpha_{\text {atm }}=1.0\right.$ down to $\tau_{\text {ph }}=100$ and $\left.\alpha_{\text {int }}=1.9\right)$ are cooler than ATLAS9 tracks with $\alpha_{\text {atm }}=0.5$ down to $\tau_{\mathrm{ph}}=10$ and $\alpha_{\text {int }}=2.3$. But Fig. 5 shows that $T_{\text {eff }}$ values as low as in NextGen models can be obtained using the ATLAS9-tracks with $\alpha_{\mathrm{atm}}=0.5, \tau_{\mathrm{ph}}=100$ and $\alpha_{\mathrm{int}}=6.3$. The reason is just that, when we integrate the atmosphere down to $\tau_{\mathrm{ph}}=100$, we adopt a lower efficiency of convection in a larger region, and so the $T_{\text {eff }}$ of the model decreases.

We computed stellar models for AH97 BCs with $\alpha_{\text {int }}=1$ and $\alpha_{\text {int }}=1.9$, for three different choices of $\tau_{\mathrm{ph}}(3,10$ and 100). As in the FST case (Sect. 4), when we use $\alpha_{\text {int }}=1.0$, the same as in the atmosphere models, there is only a small difference between evolutionary tracks whose interior has been integrated until $\tau_{\mathrm{ph}}=3,10$, or 100 . The difference between $\tau_{\mathrm{ph}}=3$ 


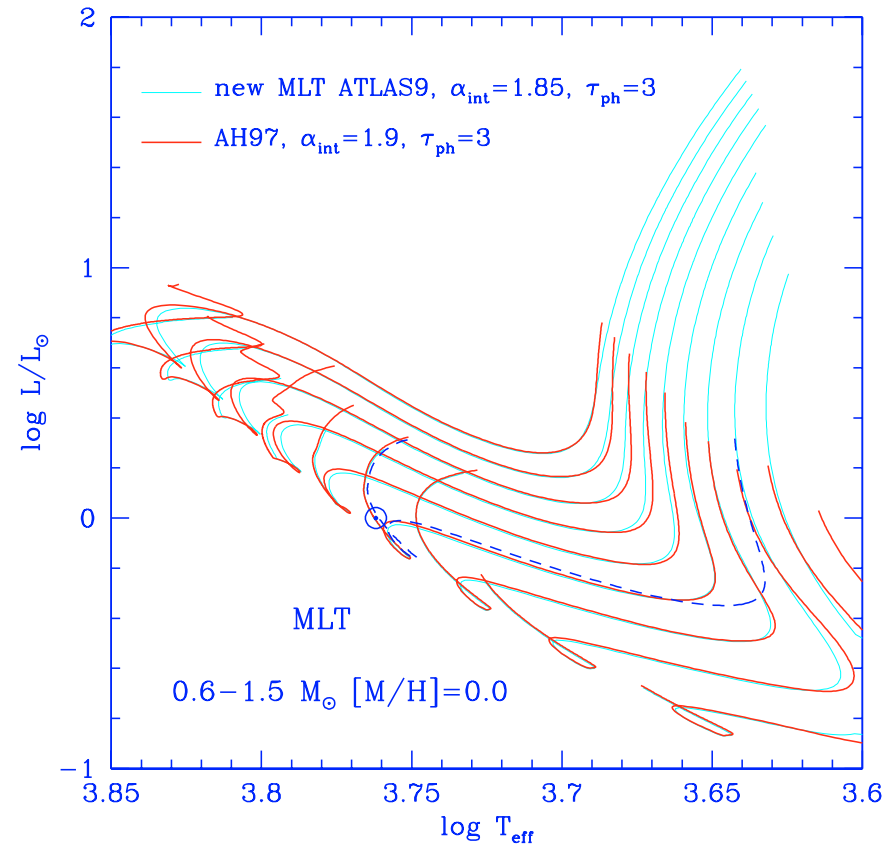

Fig. 10. Comparison between MLT models using ATLAS9 atmospheres and AH97 atmospheres until $\tau_{\mathrm{ph}}=3$. In both cases the parameters are chosen to fit the Sun. The dashed-line is a $1 M_{\odot}$ evolutionary track computed with $\alpha_{\text {int }}=1.9$ and with AH97 atmospheres until $\tau_{\mathrm{ph}}=100$.

and $\tau_{\mathrm{ph}}=100$ tracks, due to the incoherence introduced by the diffusion approximation used in the interior integration, is of the order of $40 \mathrm{~K}$ in the low-gravity domain. When we use $\alpha_{\text {int }}=1.9$, but the atmospheric grids still have $\alpha=1.0$, the PMS track location depends as well on $\tau_{\mathrm{ph}}$. Thus, the $\tau_{\mathrm{ph}}=3$ tracks are now $\sim 170 \mathrm{~K}$ hotter than the $\tau_{\mathrm{ph}}=100$ tracks (see Fig. 10 for the relative location of $1 M_{\odot}$ tracks, thick-solid line and dashed line).

In Fig. 10 we plot the evolutionary tracks calibrated on the Sun and integrating the internal structure up to $\tau_{\mathrm{ph}}=3$ for both atmosphere models, NextGen and new MLT ATLAS9 models. The overlap of both sets of models for the whole common $T_{\text {eff }} / \log g$ domain is, at first sight, surprising: it points out the similarity of the opacity in the atmosphere models (NextGen versus ATLAS9) in this domain. Generally, at $\tau=3$ the gradient of temperature still follows the radiative gradient of temperature, and hence the convective fluxes and the choice of $\alpha_{\text {atm }}$ do not have a relevant role.

These results confirm that for $T_{\text {eff }} \geq 4000 \mathrm{~K}$ the convection model is much more important than the contribution of additional molecules to opacity already included in ATLAS9 (as concluded by DM94 on the basis of grey models).

\subsection{FST versus $M L T$}

The main difference between FST and MLT that we must keep in mind is that in the region of low efficiency FST is much less efficient, while, in the high efficiency region, the FST convective fluxes are $~ 10$ larger than the MLT ones, so FST describes a much more efficient convection in deep layers. This means that, deep in the atmosphere, the MLT models yield

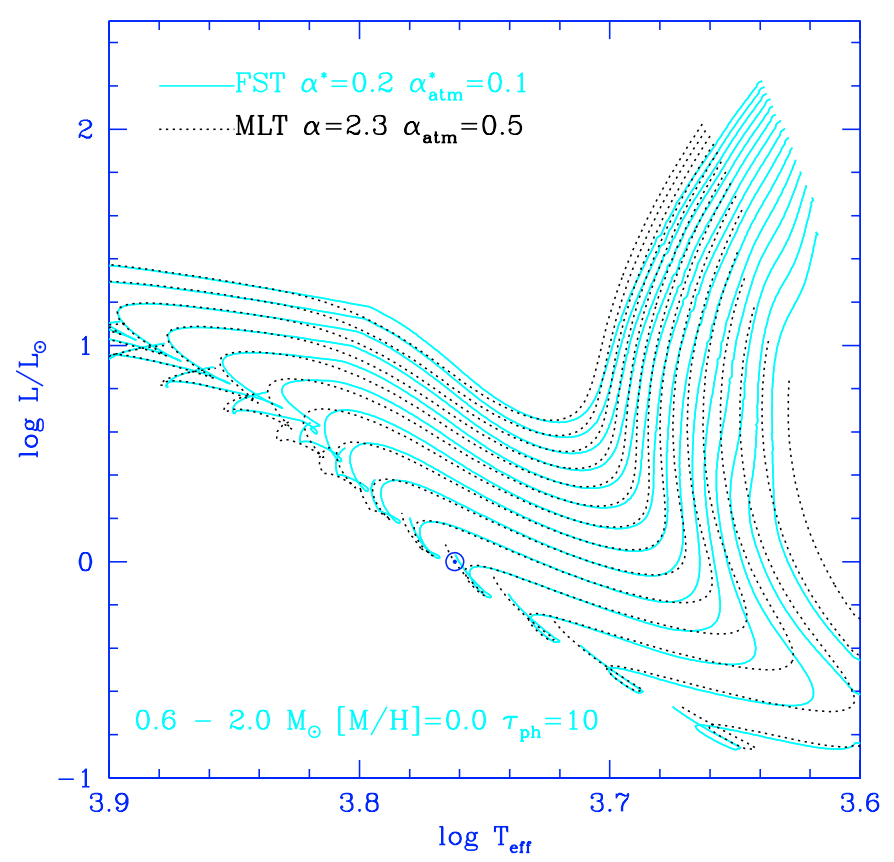

Fig. 11. PMS tracks obtained using parameters that fit the Sun, for FST and MLT treatment of convection using the new MLT and FST ATLAS9 atmosphere models.

higher temperature gradients than the FST ones (cf. Fig. 6 for $\log (P)>5.3$ ), and hence lower $T_{\text {eff }}$. DM97, 98 showed that, when grey BCs are used, the difference in $T_{\text {eff }}$ between FST and MLT tracks for solar mass tracks fitting the Sun was in between $5-10 \%\left(\sim 250 \mathrm{~K}\right.$ for $\left.1 M_{\odot}\right)$. This difference is relatively small in absolute value, but quite large, if we consider that PMS tracks of different masses are nearly parallel and rather close to each other. Thus, the masses of TTauri determined by comparison between their location on the HRD and either the MLT or FST evolutionary tracks, could differ up to $50 \%$.

If non-grey BCs are used, the $T_{\text {eff }}$ location of MLT tracks also depends on $\tau_{\mathrm{ph}}$ and therefore a direct comparison with FST tracks is not obvious. Figure 11 shows the new FST and MLT Hayashi tracks computed with the parameters $\left(\alpha^{*}\right.$ and $\left.\alpha\right)$ calibrated on the Sun and $\tau_{\mathrm{ph}}=10$. We note that the differences increase as the stellar mass decreases, and they are almost negligible for $M \geq 1 M_{\odot}$. There are two reasons that explain why the MLT and FST tracks are now so close to each other: $i$ ) Figure 1 shows that there is a domain in $\left(T_{\text {eff }}, \log g\right)$ where both sets of atmosphere models with low convection efficiency provide very similar atmospheric structures for $\tau \leq 10$, and hence similar BCs. ii) The low efficiency of MLT convection below $\tau=10$ is more or less compensated by a higher value of $\alpha$ (2.3) in the interior. The total effect is such that the largest differences in $T_{\text {eff }}$ are of the order of $100 \mathrm{~K}$ $(\sim 2 \%)$, that induce an uncertainty in the mass determination of observed PMS stars between 10-17\%.

Note also the difference between FST and MLT MS models at $\sim 6500 \mathrm{~K}$, where convection in the atmosphere is highly inefficient (this can be expected from their different atmospheric temperature structure as shown by Fig. 1, see also D'Antona et al. 2002). 


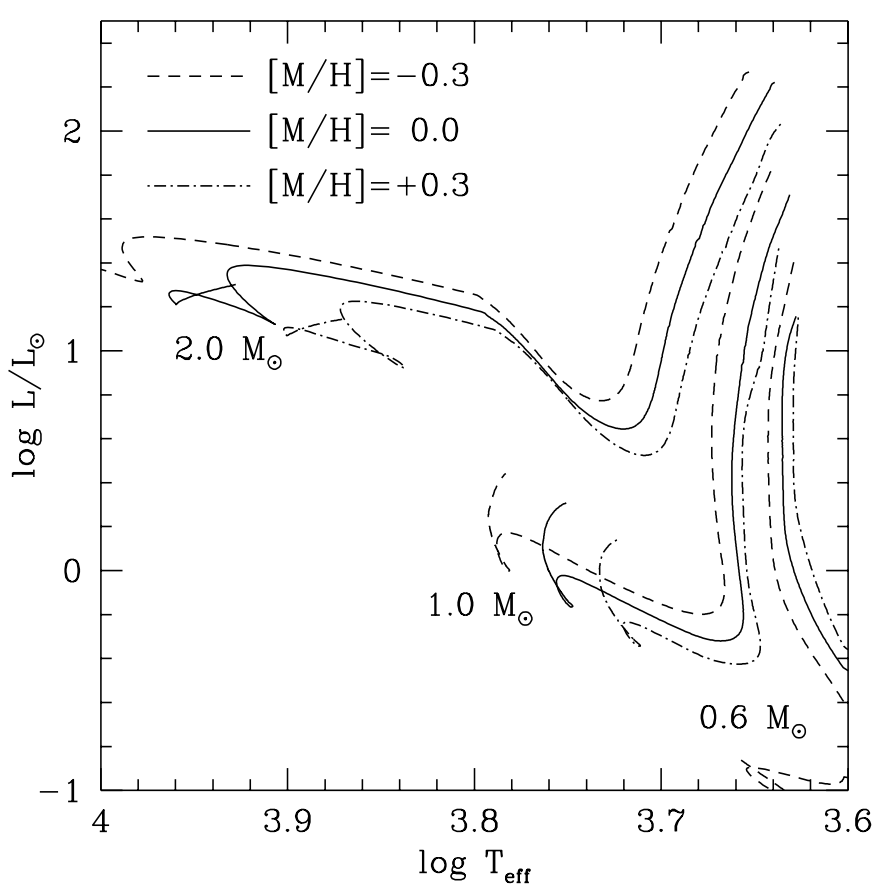

Fig. 12. PMS tracks of $2.0,1.0$, and $0.6 M_{\odot}$ stars for three different chemical compositions $([\mathrm{M} / \mathrm{H}]=-0.3,0.0,+0.3)$. All these tracks have been computed using as boundary conditions the new ATLAS9 (CGM, $\alpha^{*}=0.1$ ) atmosphere models at $\tau_{\mathrm{ph}}=10$ and the CGM formalism for the convection in the interior with $\alpha^{*}=0.2$.

\section{Metallicity and PMS location}

We have computed FST-based models also for two other metallicities, $[\mathrm{M} / \mathrm{H}]=-0.3$ and +0.3 . All of them included the CGM convection model with $\alpha^{*}=0.1$ until $\tau_{\mathrm{ph}}=10$, and $\alpha^{*}=0.2$ for the layers below. Figure 12 shows that changes of the chemical composition by a factor 2 with respect to the solar one produce a small effect on the $T_{\text {eff }}$ location of PMS tracks for FST models: $\sim 100 \mathrm{~K}$ hotter for $[\mathrm{M} / \mathrm{H}]=-0.3$ and $\sim 100 \mathrm{~K}$ cooler for $[\mathrm{M} / \mathrm{H}]=+0.3$. In Fig. 13 we compare our FST models with MLT models by Siess et al. (2000) for $1 M_{\odot}$. At $\log L / L_{\odot}=0.6$, the FST-based model with $[\mathrm{M} / \mathrm{H}]=-0.3$ is $\sim 130 \mathrm{~K}$ hotter than that one with solar composition, while MLT models differ by $\sim 170 \mathrm{~K}$. Note that in the Siess et al. (2000) models the $Z=0.02$ solar track does not

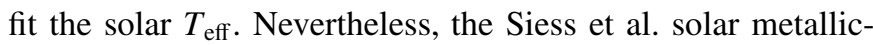
ity $(Z=0.02)$ PMS models, being cooler, do not make a bad job in reproducing the observations, according to the analysis by Simon et al. (2000).

\section{Comparison with the observations}

The theoretical uncertainties in the location of the PMS, confirmed in the present computation, are such that direct mass determinations of PMS are needed to constrain the models. In particular, the eclipsing double-lined spectroscopic binaries allow simultaneous determination of masses and radii of the components. Only one binary of this type is known by now to contain two PMS components, namely RX J0529.4+0041 (Covino et al. 2000) while several other PMS spectroscopic double-lined non-eclipsing binaries (Melo et al. 2001) can just

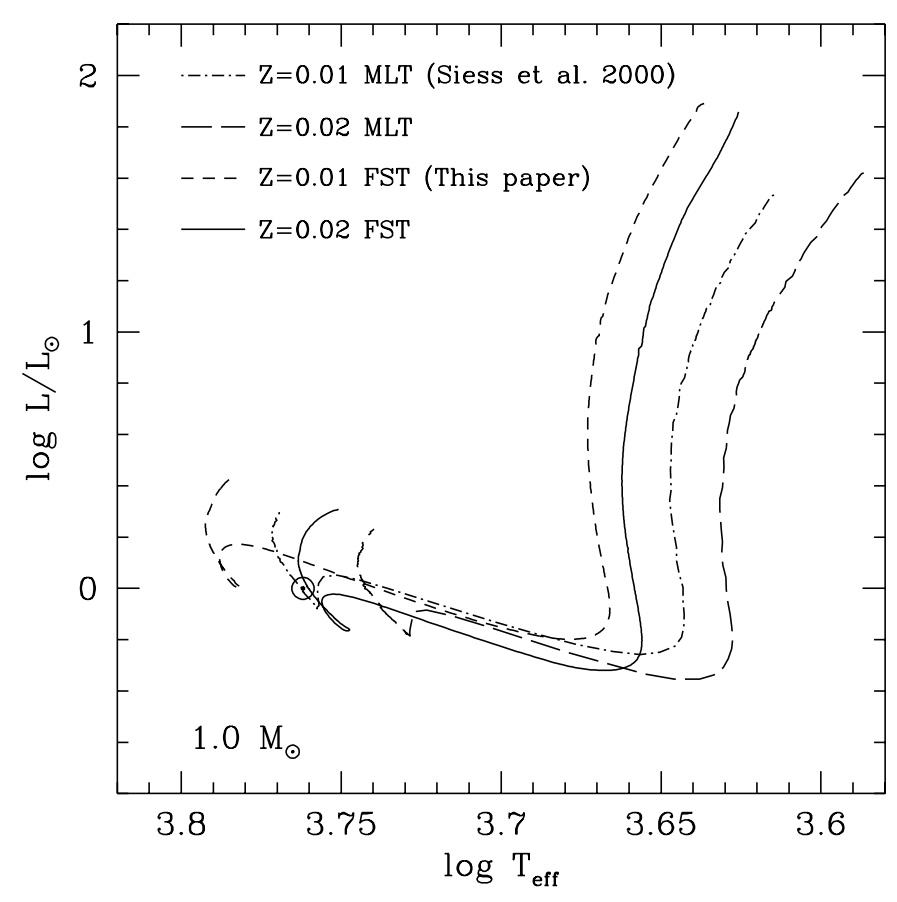

Fig. 13. Evolutionary tracks for $1 M_{\odot}$ for metallicity $Z=0.01$ and $Z=0.02\left(Z_{\odot}\right)$ and two treatments of convection: FST with $\alpha^{*}=0.2$ and MLT from Siess et al. (2000).

constrain the minimum mass of the components. In Fig. 14 we plot the masses derived by comparing the observationally derived $T_{\text {eff }}$ (and luminosity) with the theoretical evolutionary FST-tracks versus the dynamical masses. The error bar on the theoretical mass reflects the observational error on the temperatures quoted by the authors $(\sim 100-200 \mathrm{~K})$. A very small error is generally attributed to the dynamical masses. Apart from the case of RX J 0529.4+0041, for which the inclination $i$ is known, the plotted dynamical mass is the minimum mass $M \sin ^{3} i$. Many points remain below the diagonal line in Fig. 14, and the discrepancy could be larger if we had a precise indication of an $i<90^{\circ}$. This result, although still preliminary in view of the very few observational points and of the uncertainty of $T_{\text {eff }}$ determination of the PMS objects, indicates that even with the inclusion of non-grey FST model atmospheres, the FST convection is too efficient to describe the PMS. Moreover it confirms the preliminary comparisons based on grey FST models by Simon et al. (2000) and Covino et al. (2001). It is possible that the error bars on $T_{\text {eff }}$ as provided by the authors were underestimated. In order to make the mass derived for the coolest component of RX J0529.4+0041 compatible with observations, the error bar should grow up to $\sim 400 \mathrm{~K}$. Since the PMS-FST tracks are very close to each other in $T_{\text {eff }}$ (at $\log L / L_{\odot}=0.3, \Delta T_{\text {eff }}$ between 1.5 and $0.6 M_{\odot}$ is only $\sim 650 \mathrm{~K}$ ), such a large error in $T_{\text {eff }}$ also for the other stars would imply, however, that no useful constraint on the models could be obtained from this comparison.

Baraffe et al. (2002) found that most masses in PMS binaries are better reproduced with $\alpha_{\text {int }}=1.9$, but some others require $\alpha_{\text {int }}=1$, while in principle there is no physical reason to justify this difference in the efficiency of convection in the same region of the HR diagram. In fact, we have seen in Sect. 5 


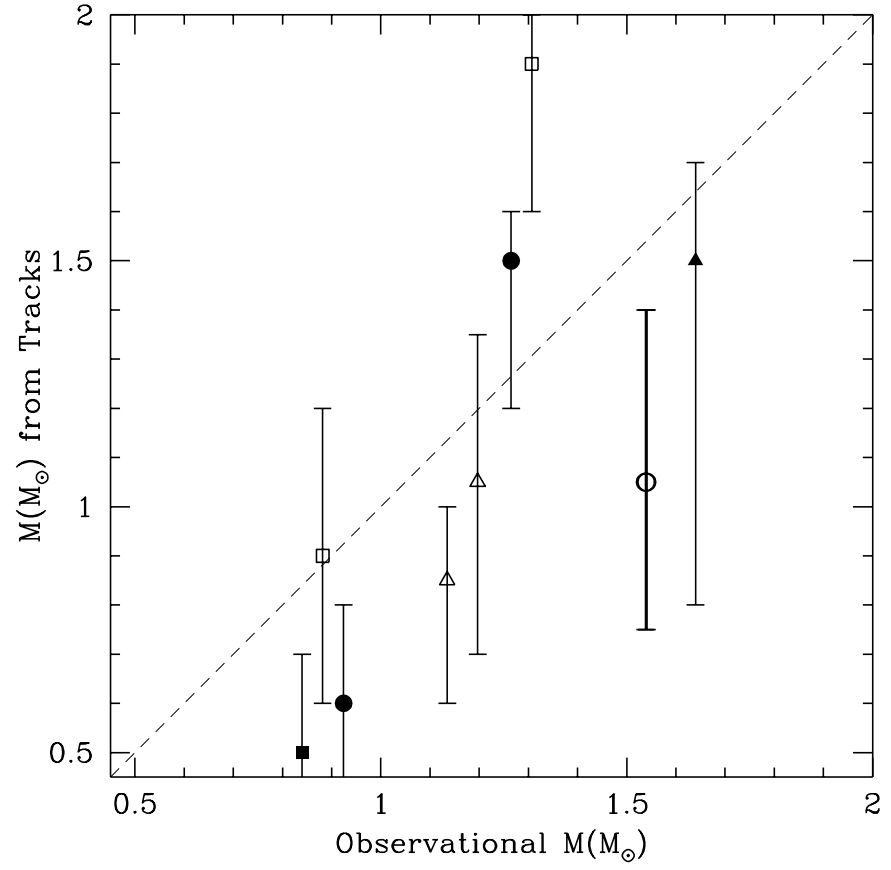

Fig. 14. The mass determined from the HR diagram position with respect to the evolutionary FST tracks is plotted with respect to the dynamical mass for the double lined spectroscopic and eclipsing binary RX J0529.4+0041 (full circles); the other points are for the double lined spectroscopic binaries RX J0541.4-0324 (empty squares); RX J0532.1-0732 (empty triangles); RX J0530.7-0.434 (empty circle, only one because $M, T_{\text {eff }}$ and $L$ are the same for both stars), in which the minimum dynamical mass is plotted. The data are from Covino et al. (2001). In addition, the location of TYCrA (full triangle) (Casey et al. 1998) and GMAur (full square) (Dutrey et al. 1998) is shown.

(Fig. 9) that for a given mass, the MLT tracks may differ even by $500 \mathrm{~K}$ if we consider both models with small and large $\alpha$ and disregard any solar constraint. Furthermore, for solar calibrated models, a variation of $\tau_{\text {ph }}$ from 3 to 100 could produce a displacement of their PMS tracks of the order of $200 \mathrm{~K}$ (compare Fig. 9 with Fig. 10). No progress in understanding is possible with this philosophy. In particular, there may be hidden parameters which affect the tracks location that could not be isolated unless we can - at least in part - falsify the models.

FST, contrarily, does not allow large displacements on the HRD by changing the parameters. The FST convection has also been shown to be very useful both to describe the solar structure (see the introduction) and many other different evolutionary phases (e.g. the DB white dwarfs pulsation boundary Benvenuto \& Althaus 1997 -, the Hot Bottom Burning phase in the intermediate mass Asymptotic Giant Branch stars -Ventura et al. 2001, 2002-, the fast transition between deep and atmospheric convection in main sequence stars -D'Antona et al. 2002): this may lead us to suspect that there is some other physical input specific of PMS models, which modifies convection in the PMS stars. Just as an example, we notice that GM Aur, which has a dynamical mass estimation of $0.84 \pm 0.05 M_{\odot}$ (Dutrey et al. 1998) and an evolutionary mass of $0.5 \pm 0.2 M_{\odot}$ also shows a magnetic field of $4-5 \times 10^{3}$ Gauss (Johns-Krull et al. 1999). D'Antona et al. (2000) have shown that the purely thermal effect of a magnetic field deeply modifies the behavior of atmospheric convection, precisely in the required direction of enlarging the temperature gradients and lowering the models $T_{\text {eff }}$.

\section{Summary and conclusions}

The new ATLAS9 atmosphere models based on the CGM treatment of convection have allowed us to compute, for $T_{\mathrm{eff}}>$ $4000 \mathrm{~K}$ and three different metallicities, new grids of nongrey FST-PMS evolutionary tracks with the same convection treatment in the interior and in the atmosphere. They provide a significant improvement with respect to DM97,98 grey FST-PMS tracks. Furthermore, we have examined one by one the parameters affecting the $T_{\text {eff }}$ location of PMS tracks analyzing the "traps" hidden in the modeling of convection in PMS and taking advantage of the use of new grids of stellar atmospheres constructed with different assumptions for convection.

Concerning the choice of the match point $\tau_{\mathrm{ph}}$ between the atmosphere and the interior, we have pointed out that the differences between the micro-physics (opacity tables and equation of state) inputs in the interior and the atmosphere models introduce a negligible uncertainty on the derived HRD location. Actually, the spatial resolution of the deepest layers of the atmosphere model is much more relevant for the choice of $\tau_{\mathrm{ph}}$. We have seen that low resolution models could induce nonnegligible errors in the BCs, especially if $\tau_{\mathrm{ph}}=100$ is taken as the match point between atmosphere and interior. This choice may otherwise appear more suitable due to the flatter temperature gradient and the ascertained validity of the diffusion approximation of radiative transfer at this depth.

We show that, while a successful solar calibration of a $1 M_{\odot}$ model adopting a low efficiency of convection in the atmosphere can be achieved by adequately increasing the efficiency of convection in the interior, sets of parameters which allow obtaining a good solar radius do not provide the same PMS tracks. In fact, different couples of parameters $\left(\alpha_{\mathrm{int}}, \tau_{\mathrm{ph}}\right)$ introduce a dispersion in the $T_{\text {eff }}$ of the PMS track of $1 M_{\odot}$ of the order of $200 \mathrm{~K}$ (if we take into account also the FST track, the uncertainty on $T_{\text {eff }}$ grow up to $250 \mathrm{~K}$ ). Since there is no physical reason to have a different efficiency of convection at each side of a fixed $\tau_{\mathrm{ph}}$, the introduction of another parameter reduces significantly the predictive power of such models: in non-grey stellar models computed using different convection in the interior and in the atmosphere, the parameters are at least three: $\alpha_{\mathrm{int}}, \alpha_{\mathrm{atm}}$ and $\tau_{\mathrm{ph}}$.

While in the MLT models the value $\alpha_{\text {atm }}=0.5$ is chosen to fit the Balmer lines, in the CGM ones, the fine tuning parameter $\alpha^{*}$ cannot be constrained by the spectral features of solar type stars, since $\alpha^{*}$ affects mainly the gradients in the deep layers of the solar atmosphere. The value adopted from the grey solar calibration by Canuto et al. (1996) turns out to be too low for the non-grey model of the Sun, and a variation of $\alpha_{\text {int }}^{*}$ of a factor of two with respect to $\alpha_{\text {atm }}^{*}$ is required. A solar model with exactly the same convection treatment in the interior and in the atmosphere should adopt an intermediate value of $\alpha^{*}$. Nevertheless, by changing $\alpha_{\text {int }}^{*}$ within this range we are 
not able to produce significant displacements over the HR diagram, and the $\Delta T_{\text {eff }}$ on the PMS tracks due to this change is smaller than $100 \mathrm{~K}$ for $M<1.5 M_{\odot}$.

The evolutionary track using grey atmospheres and a MLT treatment of convection with a $\alpha\left(T_{\text {eff }}, \log g\right)$ value provided by the 2D-model calibration (Ludwig et al. 1999) is quite close (in a large $\log g / T_{\text {eff }}$ domain) to that one obtained with a non-grey FST model fitting the Sun. This implies that the specific entropy jump between the photosphere and the adiabatic zone is almost the same in the FST based models and in the 2D-hydrodynamical atmosphere models. However, one should expect the specific entropy jump and hence PMS evolutionary tracks obtained from a 3D-hydrodynamical model atmosphere calibration to be different. As shown by Asplund et al. (2000), for solar surface convection numerical simulations in $2 \mathrm{D}$ yield higher temperatures in the deeper layers and lower ones in optically thin regions when compared to their $3 \mathrm{D}$ counterparts (see their Fig. 9). The calibration by Trampedach et al. (1999) which is based on such 3D numerical simulations cannot be used safely to calculate PMS evolutionary tracks, because the model calculations used to derive the coefficients of their fitting functions are limited to essentially the main sequence band. Ludwig et al. (1999) explicitly warn their readers that their fits quickly loose their meaning outside the region covered by their own model grid. Fortunately, their grids have been computed for a much wider region of the HRD which in fact is just large enough to include the solar PMS track as shown in Fig. 5 within the domain valid for their calibration.

We obtain quite similar PMS tracks in some regions of the HRD by using FST or MLT $\left(\alpha_{\mathrm{atm}}=0.5, \alpha_{\mathrm{int}}=2.3, \tau_{\mathrm{ph}}=10\right)$ stellar models. The reason is that MLT $(\alpha=0.5)$ and FST atmospheres have $(P, T)$ structures which overlap for optical depths smaller than $\tau \sim 10$, and the higher efficiency of FST at larger optical depths is compensated by a larger value of the mixing length parameter. The maximum $\Delta T_{\mathrm{eff}}$ is of the order of $100 \mathrm{~K}$, for the lowest considered masses.

The comparison between AH97 and the new ATLAS9-based models shows that: i) both sets of models are equivalent if the match point between atmosphere and internal structure is chosen not too deep in the atmosphere (e.g. $\tau_{\mathrm{ph}}=3$, Fig. 10). Since in this region the temperature gradient follows the radiative gradient, and the radiative gradient is determined mainly by the opacity, the overlapping between both sets of models all over the $T_{\text {eff }}-\log g$ domain considered allows us to conclude that the improvement of opacity in AH97 models has no relevant role for the location of stellar evolution tracks in this region of the HR diagram; ii) the differences between MLT-ATLAS9 models and AH97 models are only due to the specific treatment of the over-adiabatic convection. Therefore, at $T_{\mathrm{eff}} \geq 4000 \mathrm{~K}$ the convection model is much more important than the contribution of additional molecules to the opacity already included in ATLAS9 (as concluded by DM94 on the basis of grey models).

Examining some PMS binaries, which provide independent determination of the stellar masses, the new FST $\tau_{\mathrm{ph}}=10$ models provide an on average too efficient convection with respect to the masses and HR diagram location of these binaries. Covino et al. (2001) and Steffen et al. (2001) found that the best fit is obtained when MLT with $\alpha=1$ (in the interior and in the atmosphere) is adopted. Since MLT based models must adopt a value of $\alpha$ larger than 1 to fit the Sun, and since FST treatment (that provides a quite efficient convection) is able to fit the solar radius, the comparison with observation suggests that convection in the PMS evolutionary phase seems to be less efficient than for the Sun. The FST model cannot be tuned too much, but it nevertheless provides very valuable results for many different evolutionary phases. Therefore, instead of dismissing the model at this point, we suggest that there may still be another physical parameter affecting the location of PMS tracks in the HR diagram. Processes such as, e.g., interaction with a circumstellar disk (Flaccomio et al. 2003), acceleration and/or braking of rotational velocity during PMS evolutionary phase, or presence of a dynamo magnetic field (Ventura et al. 1998b; D' Antona et al. 2000) could modify the convective temperature gradients in the outer layers of PMS stars.

Finally, also the sub-photospheric structures of the Sun, as obtained by different choices of BCs and convection modeling, are quite different. The main effect of these different temperature gradients is a change in the acoustic cut-off frequency that leads to a different high frequency p-mode spectrum. Thus, the FST-based model reproduces the solar spectrum better than the MLT one does. While this improvement in the agreement with the measured mode frequencies does not imply that the model is actually "right", since other uncertainties in the physics such as atmospheric opacity, radiative transfer, etc. should be considered, a model which fits a smaller number of observational data appears, from a pragmatic point of view, less acceptable. There are, however, other aspects of surface convection in the Sun that cannot be fitted with a local 1D model such as FST, as discussed in Sects. 3.2.1 and 4.1 and in references cited therein. Major progress towards non-local and non-homogeneous models of convection suitable for evolutionary track computations is thus urgently needed to improve current stellar models.

Acknowledgements. J.M. and F.D. acknowledge support from the Italian Space Agency ASI under the contract ASI I/R/037/01. J.M. also acknowledges the support of Osservatorio Astronomico di Roma. F.K. acknowledges support by the Fonds zur Förderung der wissenschaftlichen Forschung (project P13936-TEC) and by the UK Particle Physics and Astronomy Research Council under grant PPA/G/O/1998/00576. U.H. is supported by NSF grant AST-0086249.

\section{References}

Alexander, D. R., \& Ferguson, J. W. 1994, ApJ, 437, 879

Allard, F., \& Hauschildt, P. 1997 (AH97) NextGen, http://dilbert. physast.uga.edu/ yeti/mdwarfs.html Asplund, M., Ludwig, H.-G., Nordlund, Å., \& Stein, R. F. 2000, 359, 669

Baraffe, I., \& Chabrier, G. 1997, A\&A, 327, 1039

Baraffe, I., Chabrier, G., Allard, F., \& Hauschildt, P. H. 1995, ApJ, 446, L35

Baraffe, I., Chabrier, G., Allard, F., \& Hauschildt, P. 1998, A\&A, 337, 403 (BCAH98)

Baraffe, I., Chabrier, G., Allard, F., \& Hauschildt, P. 2002, A\&A, 382, 563

Baturin, V. A., \& Mironova, I. V. 1995, AZh 72, 120, L65

Benvenuto, O. G., \& Althaus, L. G. 1997, MNRAS, 288, 1004 
Bernkopf, J. 1998, A\&A, 332, 127

Böhm, K. H., \& Stückl, E. 1967, Z. Astrophys., 66, 487

Böhm-Vitense, E. 1958, Z. Astrophys., 46, 108

Canuto, V. M. 1992, ApJ, 392, 218

Canuto, V. M. 1996, ApJ, 467, 385

Canuto, V. M., \& Mazzitelli, I. 1991, ApJ, 370, 295

Canuto, V. M., Goldman, I., \& Mazzitelli, I. 1996, ApJ, 473, 550

Casey, B. W., Mathieu, R. D., Vaz, L. P. R., Anderson, J., \& Suntzeff, N. B. 1998, AJ, 115, 1617

Castaing, B., Gunaratne, F., Helsot, F., et al. 1989, J. Fluid Mech., 204, 1

Castelli, F. 1996, in Model Atmospheres and Spectrum Synthesis, ed. S. J. Adelman, F. Kupka, \& W. W. Weiss, ASP Conf. Ser., 108, 85

Castelli, F., Gratton, R. G., \& Kurucz, R. L. 1997, A\&A, 318, 841

Christensen-Dalsgaard, J. 1997, in SCORe'96: Solar convection and oscillations and their relationship, ed. F. P. Pijpers, J. Christensen-Dalsgaard, \& C. S. Rosenthal (Kluwer Academic Publisher), 3

Covino, E., Catalano, S., Frasca, A., et al. 2000, A\&A, 361, 49

Covino, E., Melo, C., Alcalá, J. M., et al. 2001, A\&A, 375, 130

Cox, J. P., \& Giuli, R. T. 1968, Principes of Stellar Strucure (New York: Gordon and Breach), Chap. 14

D'Antona, F. 2000, Star formation from the small to the large scale, ESLAB Symp. (33: 1999: Noordwijk, The Netherlands), ed. F. Favata, A. Kaas, \& A. Wilson, ESA SP 445, 161

D’Antona, F., \& Mazzitelli, I. 1994, ApJS, 90, 467 (DM94)

D'Antona, F., \& Mazzitelli, I. 1997, Mem.S.A.It., 68, 807 (DM97)

D'Antona, F., \& Mazzitelli, I. 1998, in Brown dwarfs and extrasolar planets, ed. R. Rebolo, E. L. Martin, \& M.-R. Zapatero Osorio, ASP Conf. Ser., 134, 442 (DM98)

D'Antona, F., Ventura, P., \& Mazzitelli, I. 2000, ApJ, 543, L77

D’Antona, F., Montalbán, J., Kupka, F., \& Heiter, U. 2002, ApJ, 564, L93

Dutrey, A., Guilloteau, S., Prato, L., et al. 1998, A\&A, 338, L6

Flaccomio, E., Micela, G., \& Sciortino, S. 2003, A\&A, 402, 277

Freytag, B. 1995, Ph.D. Thesis, Univ. Kiel

Freytag, B., Ludwig, H.-G., \& Steffen, M. 1996, A\&A, 313, 497

Fuhrmann, K., Axer, M., \& Gehren, T. 1993, A\&A, 271, 451

Fuhrmann, K., Axer, M., \& Gehren, T. 1994, A\&A, 285, 585

Gardiner, R. B., Kupka, F., \& Smalley, B. 1999, A\&A, 347, 876

Gough, D. O., \& Weiss, N. O. 1976, MNRAS, 176, 589

Grevesse, N., \& Noels, A. 1993, in Origin and Evolution of the Elements, ed. N. Prantos, E. Vangioni-Flam, \& M. Cassé (Cambridge University Press), 14

Grossman, S. A. 1996, MNRAS, 279, 305

Hauschildt, P. H., Allard, F., \& Baron, E. 1999a, ApJ, 512, 377

Hauschildt, P. H., Allard, F., Ferguson, J., Baron, E., \& Alexander, D. R. 1999b, ApJ, 525, 871

Heiter, U., Kupka, F., Pauznen, E., Weiss, W. W., \& Gelbmann, M. 1998, A\&A, 335, 1009

Heiter, U., Kupka, F., van’t Veer-Menneret, C., et al. 2002a, A\&A, 392, 619

Heiter, U., Kupka, F., van't Veer-Menneret, C., et al. 2002b, in Modeling of Stellar Atmospheres, ed. N. E. Piskunov, W. W. Weiss, \& D. F. Gray, IAU Symp., 210, Uppsala 17-21 June

Henyey, L. G., Vardya, M. S., \& Bodenheimer, P. 1965, ApJ, 142, 841
Itoh, N., \& Kohyama, Y. 1993, ApJ, 404, 268

Itoh, N., Mutoh, H., Hikita, A., \& Kohyama, Y. 1992, ApJ, 395, 622

Johns-Krull, C. M., Valenti, J. A., \& Koresko, C. 1999, ApJ, 516, 900

Kupka, F., \& Montgomery, M. H. 2002, MNRAS, 330, L6

Kurucz, R. L. 1993, ATLAS9 Stellar Atmosphere Programs and $2 \mathrm{~km} \mathrm{~s}^{-1}$ grid (Kurucz CD-ROM No 13)

Kurucz, R. L. 1998, http: //cfaku5.hardvard.edu/

Ludwig, H.-G., Freytag, B., \& Steffen, M. 1999, A\&A, 346, 111

Maeder, A., \& Meynet, G. 1991, A\&AS, 89, 451

Mazzitelli, I., \& Moretti, M. 1980, ApJ, 235, 955

Melo, C. H. F., Covino, E., Alcalá, J. M., \& Torres, G. 2001, A\&A, 378, 898

Montalbán, J., D’Antona, F., \& Mazzitelli, I. 2000, A\&A, 360, 935

Montalbán, J., Kupka, F., D’Antona, F., \& Schmidt, W. 2001, A\&A, 370,982

Montalbán, J., D’Antona, F., \& Kupka, F. 2002, in Modeling of Stellar Atmospheres, ed. N. E. Piskunov, W. W. Weiss, \& D. F. Gray, IAU Symp., 210, Uppsala 17-21 June

Monteiro, M. J. P. F. G., Christensen-Dalsgaard, J., \& Thompson, M. J. 1995, in GONG '94: Helio- and Astero-Seismology from the Earth and Space, ASP Conf. Ser., 76, 128

Morel, P., van't Veer-Menneret, C., Provost, J., et al. 1994, A\&A, 286, 91

Prandtl, L. 1925, Zs. Angew. Math. Mech. 5 (N-2), 36

Rogers, F. J., \& Iglesias, C. A. 1996, ApJ, 464, 943

Rogers, F. J., Swenson, F. J., \& Iglesias, C. A. 1996, ApJ, 456, 902

Schlattl, H., Weiss, A., \& Ludwig, H.-G. 1997, A\&A, 322, 646

Siess, L., Dufour, E., \& Forestini, M. 2000, A\&A, 358, 593

Simon, M., Dutrey, A., \& Guilloteau, S. 2000, ApJ, 545, 1034

Smalley, B., \& Kupka, F. 1997, A\&A, 328, 349

Smalley, B., Gardiner, R. B., Kupka, F., \& Bessell, M. S. 2002, A\&A, 395, 601

Schmidt, W. 1999, Master's Thesis (Johannes Kepler University Linz, Austria)

Steffen, M., \& Ludwig, H.-G. 1999, in Theory and Test of Convection in Stellar Structure, ed. A. Giménez, E. F. Guinan, \& B. Montesinos, ASP Conf. Ser., 173, 217

Steffen, A. T., Mathieu, R. D., Lattanzi, M. G., et al. 2001, AJ, 122, 997

Trampedach, R., Stein, R. F., Christensen-Dalsgaard, J., \& Nordlund, A. 1999, in Theory and Test of Convection in Stellar Structure, ed. A. Giménez, E. F. Guinan, \& B. Montesinos, ASP Conf. Ser., 173,233

Turck-Chieze, S., Cahen, S., Casse, M., \& Doom, C. 1988, ApJ, 335, 415

van't Veer-Menneret, C., \& Megessier, C. 1996, A\&A, 309, 879

van't Veer-Menneret, C., Bentolila, C., \& Katz, D. 1998, Contributions of the Astronomical Observatory Skalnate Pleso, 27, No. 3, 223

Ventura, P., Zeppieri, A., Mazzitelli, I., \& D’Antona, F. 1998a, A\&A, 334, 953

Ventura, P., Zeppieri, A., Mazzitelli, I., \& D’Antona, F. 1998b, A\&A, 331,1011

Ventura, P., D’Antona, F., Mazzitelli, I., \& Gratton, R. 2001, ApJ, 550, L65

Ventura, P., D’Antona, F., \& Mazzitelli, I. 2002, A\&A, 393, 215

Xiong, D. R. 1985, A\&A, 150, 133 